

\title{
DISCLAIMER
}

This teport was prepared as an account of work sponsored by an agency of the United States Government. Neither the United States Government nor any agency thercol, nor any of their employees, makes any warranty, express or implied, or assumes any legal liability or responsibility for the accuracy, completeness, or uselulness of any information, apparatus, product, or process disclosed, or reptesents that its use would not infringe privalely owned rights. Reference herein to any specific commercial product, process, or service by trade name, trademark, manufacturer, or otherwise does not necessarily constitute or imply its endorsement, recommendation, or favoring by the Uniled Slates Government of any agency thereal. The views and opinions of authors expressed herein do not necessatily state or reflect those of the United States Government or any agency thereor.

\section{Time-Dependent Simulations of a Compact Ignition Tokamak}

\author{
D. P. Stotler and Glenn Bateman \\ Plasma Physics Laboratory \\ PFPL--2510 \\ Princeton University \\ Princeton, New Jersey 08543 \\ DE88 011170
}

\begin{abstract}
Detailed simulations of the Compact Ignition Tokamak are carried out using a 1-1/2-D transport code. The calculations include time-varying densities, fields, and plasma shape. It is shown that ignition can be achieved in this device if somewhat better than L-mode energy confinement time scaling is possible. We also conclude that the performance of such a compact, short-pulse device can depend greatly on how the plasma is evolved to its flat-top parameters. Furthermore, in cases such as the ones discussed here, where there is not a great deal of ignition margin and the electron density is held constant, ignition ends if the helium ash is not removed. In general, control of the deuterium - tritium density is equivalent to burn control.
\end{abstract}




\section{Introduction}

In this paper we discuss transport simulations of the Compact Ignition Tokamak (CIT). ${ }^{1}$ The flat-top parameters used in this work are given in Table $\mathrm{l}$. The goal of CIT is to study an ignited plasma in a device of minimum cost; this necessarily restricts the size of the device. Despite being comparable (e.g., DIIl-D ${ }^{2}$ ) or smaller (e.g., TFTR $^{3}$ or $\mathrm{JET}^{4}$ ) in size than existing experiments, CIT is expected to exhibit better performance due to the considerably higher fields and densities planned. Evaluation of the design requires significant extrapolations of the experimental data base. Given these extrapolations, we can use transport simulations to make detailed predictions about the performance of CIT. The primary assumptions involve describing the particle and thermal transport. There are also limits placed on the plasma density, $\boldsymbol{\rho}$ (ratio of the plasma energy to the magnetic energy), and fusion power that must be adhered to in exploring the operating space of CIT. Note that these simulations not only aid in the design of the machine itself and help to identify critical issues, but also provide parameters for diagnostic design. Furthermore, other theoretical groups can utilize the plasma profile and equilibrium information to perform more involved calculations (e.g., stability).

Although we focus here on CIT, the techniques and some of the results can be applied to tokamaks in general. As examples of the utility of transport codes, we point out that they are useful in analyzing and understarding experimental results,,$^{5-7}$ and have also been employed in predicting the performance of other jgnited tolamaks. ${ }^{8.9}$

In this paper, we show that the way in which the plasma is grown onto the divertor during the current ramp can have an impact on the fusion power production in CIT by way of controlling the size of the sawtooth mixing radius. This effect is important for CIT since the resistive skin time is long compared to the pulse length. For a steady-state reactor, these considerations are not likely to be as significant except to point out the utility of some form of current profile shaping. We also find that even at modest power production levels, helium ash accumulation (for constant electron density) can quench the ignition. In CIT, this may just provide a convenient form of burn control. Of course, if the density cannot be regulated in the actual experiment, some other form of active burn control may be required. In a general reactor, 
helium accumulation may be just as much of a problem. Such devices will require a scheme for temoving helium as it is formed in order to maintain an extended burn phase.

We will next briefly describe our transport code and some of the CIT simulations that have been performed previousiy. In Section II, we describe in detail the models and assumptions used in the present work. Then, in Section III we will discuss the results of two specific simulations. We will address a few sensitivity issues that were not discussed in the previous work ${ }^{10}$ in Section IV. Finally, we present our conclusions in Section V.

The code we use to perform the simulations has been described in detail elsewhere. ${ }^{t 1.12}$ In particular, we employ the 1-1/2-D BALDUR transport code. It integrates over time a set of flux-surface-averaged transport equations on a radial grid of flux surfaces; the shape of the flux surfaces is provided by a moments equilibrium code interfaced with the transport code. The equations solved describe diffusion of density, energy, and magnetic field:

$$
\begin{aligned}
& \left.\frac{\partial}{\partial t}\right|_{\xi}\left(n V^{\prime}\right\rangle=-\frac{\partial}{\partial \xi}\left(V^{\prime}\left\langle\Gamma_{\rho} \cdot \nabla \xi\right)-n V^{\prime \prime} \dot{\rho} / \rho^{\prime}\right)+V^{\prime}\langle S\rangle, \\
& \left.\frac{3}{2} \frac{\partial}{\partial t}\right|_{\xi}\left(n T V^{\prime \prime}\right)=-\frac{\partial}{\partial \xi}\left(V^{\prime}\left\langle q_{\rho} \cdot \nabla \xi\right\rangle-\frac{3}{2} \frac{n T V^{\prime} \dot{\rho}}{\rho^{\prime}}\right) \\
& +V^{\prime}\langle Q\rangle-n T\left[\dot{V}^{\prime}-\left(\dot{\rho} V^{\prime} / \rho^{\prime}\right)^{\prime}\right] \text {, } \\
& \left.\frac{\partial}{\partial t}\right|_{\xi}\left(B_{p} \rho^{\prime}\right)=\frac{\partial}{\partial \xi}\left\{\frac { \eta } { I ( 1 / R ^ { 2 } \rangle } \left[\frac{I^{2}}{\mu_{0} V^{\prime}} \frac{\partial}{\partial \xi}\left(\frac{V^{\prime} \rho^{\prime} B_{p}}{I}\left(\frac{|\nabla \xi|^{2}}{R^{2}}\right)\right)\right.\right. \\
& \left.\left.-\frac{\left\langle J_{\text {beom }} \cdot B\right\rangle}{R_{0 R E F}}-\frac{\left\langle J_{\text {boox }} \cdot B\right\rangle}{R_{0 R E F}}\right]+\dot{\rho} B_{p}\right\} \text {. }
\end{aligned}
$$

There are separate equations for the density, $n(\xi, t)$, of each ionic species; the electron density is computed from quasi-neulrality. Separate energy equations are solved for ions and electrons; the temperatures, $T(\xi, t)$, of all ionic species are taken to be equal. The quantity appearing in Eq. (3) is actually a normalized poloidal tlux gradient:

$$
B_{p} \equiv \frac{\partial \psi_{p \alpha} / \partial \rho}{2 \pi R_{0 F E F}} .
$$


In the limit of a circular cylinder geometry, this $B_{p}$ reduces to the actual poloidal magnetic field. Note that $\rho$ is a Aux-surface label defined by $\rho \equiv$ ( $\left.\psi_{\text {tor }} / \pi B_{\text {OREF }}\right)^{1 / 2} ; B_{0 R E F}$ and $R_{0 R E F}$ are a fixed reference magnetic field and rajoe radius. The equations have been transformed into a coordinate system with arbitrary flux surface label $\xi$; the toroidal (poloidal) fluxes are denoted by $\psi_{\text {tor }}\left(\psi_{\text {pol }}\right)$. The volume inside flux surface $\xi$ is $V(\xi, t) ; V^{\prime \prime}=\partial V / \partial \xi$. An overdot indicates a derivative with Iespect to time. The particle and heat fluxes are designated as $\boldsymbol{\Gamma}_{\rho}$ and $\boldsymbol{q}_{\rho}$; the subscript $\rho$ signifies that they are defined relative to surfaces of constant toroidal flux. Volume sources of particles (energy) are contained in $S(Q)$. Finally, in the magnetic diffusion equation, $\mathrm{Eq}$. (3), $R$ is the major radius, $\eta$ is the plasma resistivity, and $I(\xi)=R B_{\mathrm{T}}$, where $B_{\mathrm{T}}$ is the toroidal magnetic field. The code is able to treat beam-driven current $\left(\boldsymbol{J}_{\text {beam }}\right)$ and bootstrap current $\left(\boldsymbol{J}_{\text {boot }}\right)$. In all cases, the angle brackets denote a flux-surface average.

The full expressions for the fluxes and sources are quite complicated ${ }^{11}$ and will not be given here. Instead we will specify below the semi-empirical (anomalous) transport coefficients used to predict the level of particle and energy confinement in CIT. The present version of BALDUR is capable of treating sawtooth oscillations, ballooning modes, and bootstrap current.

The work discussed here is an extension of previous investigations; Singer et al. ${ }^{10}$ carried out the original simulations upon which these are based. One of their primary conclusions was that uncertainties in the experimentally calibrated transport model lead to significant variations in the predicted performance of CIT; this is still true in the present study. Since then, there have been numerous changes to the CIT design, the BALDUR code, and to the details of the plasma programming used in the simulations. Instead of relying on a semi-empizical transport model completely as done before, we alter the overall magnitude of the local transport coefficients to yield global confinement times between those expected for the L-mode and $\mathrm{H}$-mode ${ }^{13}$ (i.e., a confinement time twice that of L-mode; see, for example, Ref. 14) as computed using the scaling of Kaye and Goldston. ${ }^{15}$ The detailed profile and transient behavior information contained in the original transport models is retained in this manner and does have a significant impact on the simulations. The transport models we use here were calibrated against L-mode discharges; hence, we cannot use them to accurately predict performance during $\mathbf{H}$-mode operation. Instead, we view this procedure as one of enhancing an L-mode 
discharge. The previous conclusions regarding uncertainties in the transport model remain.

\section{Models and Assumptions}

We now describe the time dependence of the discharge parameters that are input to the code, as well as the transport models, the auxiliary heating scheme, and the MHD effects treated here. The bootstrap current is a recent addition to the 1-1/2-D BALDUR code; its effects will also be discussed in this paper.

\section{A. Parameters}

In Fig. 1 we show how the toroidal magnetic field, $B_{T}$; plasma current, $I_{p}$; elongation $\kappa$ and triangularity $\delta$ of the $95 \%$ toroidal flux surface; and volume-averaged electron density, $\left\langle n_{e}\right\rangle$, evolve as a function of time. The simulation starts with an almost circular shape and non-zero fields. By $t=3$ $\mathrm{sec}$, the plasma is on the divertor (at fuil elongation), and at $t=5.8 \mathrm{sec}$, all fields have reached their flat-top values (shown also in Table I). Note that the anxiliary heating extends from $t=4.8$ to $t=6.3 \mathrm{sec}$. The time evolution of the plasma shape is more clearly indicated in Fig. 2; these equilibrium flux contours are from the fast ramp case described in Sec. III.B. The design flattop is limited to five seconds in duration by resistive heating of the toroidal field coils. lery few of our simulations are still ignited at this point. So for present purposes, a longer flat-top is unnecessary. For simplicity, we do not consider the shut-down phase of the discharge here. Studies of this period in CIT are very critical, however, in order to avoid disruptions resulting from exceeding the density limit or MHD stability limits.

The magnetic field is ramped up such that the toroidal magnetic energy is increasing approximately linearly with time (toroidal magnetic field goes like the square root of time). On the other hand, the plasma current and shape parameters rise in a purely linear fashion. This particular ramp scheme is based upon work done by Pomphrey and Jardin. ${ }^{16}$ Their primary concern is with the design of the field coils used to determine and control the plasma shape. As will be discussed in Section III.B, the details of this ramp scheme can affect the plasma evolution and stability during flat-top. The resuits of 
our simulations can provide leedback that is useful in designing the actual plasma ramp scheme.

Like the current, the electron density is brought up (approximately) linearly. This strategy is based upon the empirical observation that the maximum allowable electron density is proportional to the plasma current. In particular, the Murakami - Hugill density limit ${ }^{17,18}$ is usually written as $\left\langle n_{E}\right\rangle_{M H} \propto B_{T} /\left(R_{q_{\text {gll }}}\right)$ (i.e., proportional to the total plasma current). When $\left\langle n_{e}\right\rangle_{M H}$ is expressed in $10^{20} \mathrm{~m}^{-3}, B_{T}$ is in $\mathrm{T}$, and $R$ is in $\mathrm{m}$, the constant of proportionality is of order unity. However, due to the scarcity of experimental data in elongated geometries, there is some uncertainty as to exactly what expression should be used for qeyt. The safety factor of an equivalent circular cylinder is $q_{a d}=5 a^{2} B_{T} /\left(R I_{p}\right)$, with $a$, the minor radius, and $R$ in $\mathrm{m}, B_{T}$ in $T$, and $I_{p}$ in $M A$. In extending the definition of $q_{z y}$ to elongated geometries, one generally multiplies this expression by some function of $\kappa$ and $\delta$ that is of order unity and reduces to unity in the circular limit. A popularly used expression multiplies $q_{\text {ew }}$ by $\left[1+\kappa^{2}\left(1+2 \delta^{2}\right)\right] / 2$; in this case, a constant of proportionality of 1.5 leads to $\left\langle n_{e}\right\rangle_{M H}=2.8 \times 10^{20} \mathrm{~m}^{-3}$. On the other hand, if just the original circular expression for $q_{c y}$ is employed, the limit increases to $8.9 \times 10^{20} \mathrm{~m}^{-3}$. There is some evidence ${ }^{19}$ that the higher limit may in fact be the more appropriate one. However, in the absence of an adequate model for the density limit, we have tried to remain close to the smaller value.

The sharp changes in the electron density are the result of large pellets composed of equal amounts of deuterium and tritium being shot in with velocities of $2 \mathrm{~km} / \mathrm{sec}$ (except for the first one done at $1.5 \mathrm{~km} / \mathrm{sec}$ ). The timing is such that the last one goes in during the auxiliary heating; it does not penetrate to the inner regions of the plasma. In general, pellet penetration is a problem for smaller or slower pellets once the plasma becomes energetic and dense. In between the pellets, the electron density is maintained at the constant level indicated in Fig. 1 by balanced deuterium and tritium gas puffing at the edge.

We also include two impurity species in these simulations. The first is helium; it accounts not only for the thermalized alpha particles, but also the $H e^{3}$ required for the planned auxiliary heating scheme (see Sec. II.D). For the latter purpose, we influx sufficient helium between $t=3.1$ and $t=4.7 \mathrm{sec}$ to reach the approximate concentration of $5 \%$ desired for efficient heating. The primary impurity is assumed to be carbon, consistent with the use of 
graphite tiles to cover the interior of the vacuum vessel. Initially, there is enough carbon present to yield $Z_{\text {eff }}=1.5$. We define

$$
Z_{e f f} \equiv \frac{\sum_{j} n_{j}\left\langle Z_{j}^{2}\right\rangle}{n_{e}}
$$

where the sum is over all ionic species; their densities and charges are designated as $n_{j}$ and $Z_{j}$, respectively. The angle brackets denote an average over the coronal equilibrium charge states. During pellet injection, $Z_{\text {eff }}$ drops steadily up to the initiation of auxiliary heating. From this point until $t=8.3$, we influx carbor, bringing $Z_{\text {eff }}$ back to about 1.5. Typically, $Z_{\text {eff }}$ continues to increase due to helium ash accumulation, adding as much as another 0.1 to $Z_{e / f}$. The boundary conditions for all impurities constrain the particle flux at the plasma edge. The code is set up so that except during periods of user-specified influx (or outflux), the number of impurity particles is constant.

\section{B. Transport Models}

We now give expressions for the particle and energy fluxes appearing in Eqs. (1) and (2). In this particular version of BALDUR the flux-surface label, $\xi$, is taken to be the square root of the normalized toroidal flux, $\xi=$ $\sqrt{i_{\text {tor }} / t_{\text {tor.a }}}$, where $\psi_{\text {eor,a }}$ is the toroidal flux at the plasma boundary.

The particle flux of ionic species $j$ is written as

$$
\left\langle\boldsymbol{\Gamma}_{\rho} \cdot \nabla \xi\right\rangle_{j}=\left(-D_{j} \frac{\partial n_{j}}{\partial \xi}\langle|\nabla \xi|\rangle+n_{j} v_{j}+\Gamma_{j}^{n=o}\right) \frac{\left\langle|\nabla \xi|^{2}\right\rangle}{\langle|\nabla \xi|\rangle}
$$

and we assume

$$
\begin{gathered}
D=\frac{1}{n_{e}(\xi) /\left(10^{19} m^{-3}\right)} m^{2} / \mathrm{sec}, \\
v=-2 D r / a^{2}
\end{gathered}
$$

$r$ is a generalization of the minor radial coordinate. Here, $r$ is defined as half of the width (the half-width) of a given flux surface on the midplane; it is thus a flux surface quantity. The neoclassical flux, $\Gamma_{j}^{\text {neo }}$, includes the Ware pinch for the hydrogenic components. The detailed expressions are given in Ref. 11. The inward pinch used here ${ }^{20}$ simulates the density peaking 
effect one hopes to achieve with pellet injection. ${ }^{21}$ As will be demonstrated below. the density profiles we get with this transport model are centrally peaked. particularly for cases with narrow sawtooth oscillations. Since this is beneficial to ignition, ${ }^{22}$ it might be argued that we are being overly optimistic. However. by making this (not unusual) assumption, we are abie to ignite with relatively conservative specifications on auxiliary heating and confinement time (see Sec. III.A).

The expression we use for the heat flux is

$$
\left\langle q_{\circ} \cdot \nabla \xi\right\rangle_{j}=\left(-\chi_{j} \frac{\partial}{\partial \xi}\left\langle\frac{3}{2} n_{j} T_{j}\right\rangle\langle|\nabla \xi|\rangle+\frac{3}{2} T_{j}\left\langle\Gamma_{\circ} \cdot \nabla \xi\right\rangle_{j}\right) \frac{\left\langle\left.\nabla \xi\right|^{2}\right\rangle}{\langle|\nabla \xi|\rangle},
$$

Where the subscript $j$ denotes electron or ion thermal flux. We will make use of the following two semi-empirically derived thermal diffusivities, $\chi_{j}$.

The first one was developed by Sinzer, Ku et al., ${ }^{23}$ and used in the original simulations of CIT with BALDUR. ${ }^{10}$ They actually obtained a family of scaling laws; the one we employ here is proportional to temperature. In particular,

$$
\begin{gathered}
\chi_{e}^{S K} \propto \frac{r}{R^{2} \kappa^{4} n_{e}^{0.875}}\left[\frac{P_{t h} / n_{e}^{0.95}}{\left(E_{r} r^{0.5} / q\right)^{0.8} Z_{e j f}^{0.4}}\right]\left|\frac{\partial \ln P_{t h}}{\partial \ln r}\right|^{1 / 2}, \\
\chi_{i}^{S K}=0.5 \chi_{e}^{S K}+\chi_{i}^{C H},
\end{gathered}
$$

where $P_{\text {th }}$ is the thermal pressure, and $q$ is the local MHD safety factor. The neoclassical contribution computed by Chang and Hinton ${ }^{24}$ is included as $\chi_{i}^{C H}$. Note that the expression in square brackets is approximately constant for ohrnic plasmas. Singer et al. ${ }^{23}$ determined the constant of proportionality in Eq. (8) to be $2.1 \times 10^{33} \pm 33 \%$ (all quantities in SI units) by comparison with experimental data.

We will compare resuits for the global energy confinement time, $\tau_{E}$, obtained using one of our two semi-empirical models with a reference confinement time, $T_{E, K G}$. Specifically, $\tau_{E, K G}$ is an inverse quadratic combination ${ }^{25}$ of a neo-Alcator ohmic scaling and the Kaye-Goldston ${ }^{15}$ auxiliary heated scaling:

$$
\frac{1}{\tau_{E, K G}^{2}}=\frac{1}{\tau_{N A}^{2}}+\frac{1}{\tau_{A U X}^{2}},
$$


where

$$
\begin{aligned}
& T_{N A}=7 \times 10^{-3}{\overline{n_{e}, 19}}_{19} a R^{2} q_{\mathrm{cyl}}, \\
& \tau_{A U X}=\tau_{A U X, K G} \times\left(\frac{A_{1} A_{2}}{A_{O} A_{H}}\right)^{0.25} \text {, } \\
& \tau_{A U X . K G}=3.04 \times 10^{-2} \kappa^{0.26} B_{T}^{-0.09} I_{p}^{1.24} P_{t o t}^{-0.58}\left(\bar{n}_{e, 19}\right)^{0.26} a^{-0.99} R^{1.65},
\end{aligned}
$$

and

$$
q_{c y l}=5 \frac{i^{2} B_{T}}{I_{p} R}\left[\frac{1+\kappa^{2}\left(1+2 \delta^{2}\right)}{2}\right] .
$$

Here. $\overline{n_{e}} .19$ is the line-averaged electron density in units of $10^{19} \mathrm{~m}^{-3}$. The minor and major radii in $m$ are denoted by $a$ and $R$. The magnetic field, $B_{\mathrm{T}}$. is in $\mathrm{T}$, and $I_{\mathrm{p}}$ is the plasme current in NIA. The total power, $P_{\text {tot }}$, (alpha, ohmic, and auxiliary) is in MW. The additional mass factor added to $T_{A} A . x$ represents one possible scaling for the mass dependence of the energy confinement time. ${ }^{26,27}$ In inis expression, $A_{i}$ is the mass of species $i ; 1$ and 2 refer to the main components of the plasma under consideration; D and $H$ are for deuterium and hydrogen, respectively.

We have adjusted the coefficients of the thermal transport models emplored in the simulations so that they yield confinement times between $\tau_{E, K G}$ (L-mode) and $2 \tau_{E, K G}$ (H-mode). For the Singer-Ku model, this amounted to using a coefficient 1.25 times larger than their original calibration $\left(2.1 \times 10^{33}\right)$; this is within their error bars. ${ }^{23}$

Our second transport scaling was developed by Redi, Tang, et al..$^{5}$ Since the detailed expressions for the thermal diffusivities are quite complicated, we refer the reader to the original work for more information. This model assumes profile-consistency. In particular, the electron temperature profile is taken to have a Gaussian shape determined only by the edge safety factor. .This constraint is used to derive a radial form factor for the thermal diffusivity. By comparing this expression with the predictions of the turbulent transport due to microinstabilities in an appropriate region of the plasma, the overall magnitude can be fixed. The modes focussed on are the trapped electron drift wave for $\chi_{e}$ and the toroidal ion temperature gradient driven instability for $\chi_{i}$. Depending upon the collisionality, $\chi_{e}$ can have one of two forms; for the highly collisionless case $\left(\nu_{-e}<0.15\right.$, with $\nu_{m}$ evaluated at the $q=1.5$ surface),

$$
x_{e}^{c^{\prime} l e s s} \propto P_{e, \text { tot }}^{0.8}(a)
$$


while for more collisional plasmas $\left(\nu_{-e}>0.15\right)$,

$$
\chi_{e}^{c^{\prime} a l} \propto P_{e, t a t}^{0.8}(a) .
$$

Here, $\nu_{a e}=\nu_{e g f} / \omega_{b}$, with $\nu_{e f f}$ and $\omega_{b}$ denoting the effective collision and trapped electron bounce frequencies, respectively. In the above expressions, $P_{e, t o t}(a)$ is the total power input into the electrons integrated over the plasma. Unlike the original model, the transi? "on between the two scalings is smoothed somewhat.

The contribution from the ion temperature gradient driven modes, $\chi_{i}^{\eta_{i}}$, is added on to the neoclassical expression of Chang and Hinton ${ }^{24}$ to form the $\chi_{i}$ that goes into Eq. (7). When $\eta_{i} \equiv \partial \ln T_{i} / \partial \ln \pi_{i}>1.5$ (also evaluated at the $q=1.5$ surface),

$$
\chi_{i}^{n i} \propto P_{i, \text { Lot }}^{0.8}(a),
$$

where $P_{i, c o t}$ is the total power input to the ions; otherwise, $\chi_{i}^{\eta_{i}}=0$. Again, this transition is smoothed somewhat in the present work, contrary to the procedure used in the original calibration.

The overall coefficients for these three scaling laws, $\chi_{e}^{\varepsilon^{\prime} l e u s,}, \chi_{i}^{\varepsilon^{\prime a l}}$, and $\chi_{i}^{n_{i}}$ have been adjusted in the simulations by a constant factor to yield global confinement times between $\tau_{E, K G}$ and $2 \tau_{E, K G}$ to reflect the enhanced confinement observed in diverted tokamaks.

The fundamental distinctions between the Redi-Tang model and the Singer$\mathrm{Ku}$ model lead to noticeable differences in the results despite the somewhat similar confinement times. Most notably, the Singer-Ku model scales with the plasma energy while the Redi-Tang expression increases with the power input into the plasina. When thermal transients are present (e.g., the sudden initiation of auxiliary heating), the Redj-Tang model will instantly show a degradation in confinement time. $O_{n}$ the other hand, a transport model scaling with energy content such as $\boldsymbol{x}_{e}^{S K}$ may take a while to exhibit a drop in confinement, and can in fact actually give rise to a temporary increase in $\tau_{E}$. The other way in which the two approaches differ is in the resulting temperature profile shape. We will say more about this distinction in Sec. III.B. 


\section{MHD and Neoclassical Effects}

Sawtooth oscillations in BALDUR are modelled using a Kadomtsev reconnection picture. ${ }^{22.28}$ Of course for there to be a reconnection, the safety factor on axis must be less than unity. When it is, the code periodically flattens the density and temperature profiles out to the mixing radius; there is no (direct) alteration of the extefior portion of the plasma. The value of the mixing radius is computed from the Kadomtsev theory ${ }^{28}$; it is typically 20 to $40 \%$ larger than the radius of the $q=1$ surface. Since detailed models for the perjodic trigger mechanism of the sawtooth oscillations are not available, the user is required to specify the period on input. For all of the simulations discussed here, it is fixed at 0.3 sec. ${ }^{10}$ The importance of sawtooth oscillations in CIT will be discussed in Sec. III.B.

BALDUR can also be instructed to compute the critical pressure gradient for ideal MHD ballooning modes using information from the equilibrium code. Furthermore, there is a mechanism for enhancing the thermal diffusivity on unstable flux surfaces, as was suggested by Connor, Taylor, and Turner ${ }^{20}$ and Azumi et al..$^{30}$ We refer the reader to Ref. 9 for details on the solution of the ballooning mode equation, the implementation of the enhanced transport, and the effects of low shear. The purpose of this procedure is solely to constrain the profiles to remain stable with respect to these modes; the effects on CIT will be described in Sec. IV.A.

BALDUR has been recently upgraded to include the neoclassical boot:strap current effect. Its implementation is described in more detail in Ref. 9. The expressions used can be shown to be similar to those found in Refs. 31 and 32. We will discuss its relevance to CIT in Sec. IV.C.

\section{Auxiliary Heating}

In order to reach ignition, the CIT design'. calls for 10 - $20 \mathrm{MW}$ of ion cyclotron resonance heating (ICRH). Present plans are to use $\mathrm{He}^{3}$ minority as:d tritium second harmonic healing. Since self-consistent computations of the deposition profile for ICRH are beyond the scope of the BALDUR code, we must provide heating information based upon studies done elsewhere. In order to maintain a realistic accounting of impurity species, we influx the $5 \%$ of $H e^{3}\left(n_{H e} / n_{e}=0.05\right)$ needed for efficient minority heating. 
For all of the simulations discussed here, auxiliary heating begins at $t=$ $4.8 \mathrm{sec}$ and ends at $t=6.3 \mathrm{sec}$. Although the toroidal field is changing during the first $1 \mathrm{sec}$ of this period, the resonance layer does not move appreciably $(<0.05 \mathrm{~m})$. We hold the location of the peak of the heating profile fixed throughout for simplicity (although the code is capable of modelling a moving profile ${ }^{33}$ ). The operating frequency of the antenna is assumed to be adjusted so that the peak of the heating is at the magnetic axis; this should be the optimum configuration for energy confinement.

At the densities planned for CIT, the focussing effect of the plasma on the incident ICRH waves is expected to be significant. ${ }^{34}$ The theoretically predicted shape of the profile is uncertain apart from this information. Here, we use a fixed, Gaussian-siaped deposition profile that drops to half of its central value at $r \simeq 0.15 \mathrm{~m}$.

The other information required to specify the auxiliary heating is the relative amount of power gojng into the electron and ion channels. Fortunately, this can be computed with a bit more confidence; in particular, we expect ${ }^{35}$ $P_{i} / P_{e} \sim 2 / 1-3 / 1$, where $P_{i}$ and $P_{e}$ refer to the power into the ions and electrons, respectively. We follow the work by Singer et al. ${ }^{\text {to }}$ and assume that the ions receive $75 \%$ of the input power.

Although $20 \mathrm{MW}$ should be available for CIT operation, we will restrict ourselves to a more conservative $10 \mathrm{MW}$ for all of the simulations presented here. Furthermore, we use throughout a $1.5 \mathrm{sec}$ beating time. In previous work, longer heating periods and higher power levels were employed. ${ }^{10,33}$ This limits the range of temperatures and densities that we can examine due to the finite amount of time required to heat to ignition temperatures given the expected losses. Nonetheless, we still predict that CIT will ignite.

\section{Results}

We now discuss in detail the results of two simulations performed using the models described above. The primary distinction between the two is the expression for the thermal diffusivity. The first (referred to hereafter as I) uses the Singer-Ku model, while the second (II) follows the Redi-Tang approach. The evolution of the volume-averaged electron density for $I$ is as shown in Fig. ? with a flat-top value of $\left(n_{e}\right)=3.0 \times 10^{20} \mathrm{~m}^{-3}$. For simulation II, we retain the basic form of the time evolution, but rescale everything to 
reach a maximum density of $\left\langle n_{e}\right\rangle=3.4 \times 10^{20} \mathrm{~m}^{-3}$. The higher density is needed to reach ignition with this more pessimistic transport model. Finally, we enhance the thermal transport on ballooning-unstable flux surfaces in simulation I, but not in II. We will discuss this procedure and its effect on ignition in Sec. IV.A.

\section{A. Ignition Parameters}

In Figs. 3 and 4, we show the time evolution of the different contributions to the input power for each of the two simulations. The ohmic power rises during the current ramp; the small spikes are the result of the temperature drops accompanying each pellet injection. Once the auxiliary heating is turned on and the electron temperature rises above a few $\mathrm{keV}$, the ohmic power falls; it levels off at a flat-top value of $1-2 \mathrm{MW}$.

As mentioned previously, both runs assume that $10 \mathrm{MW}$ of auxiliary power is being absorbed between $t=4.8 \mathrm{sec}$ and $t=6.3 \mathrm{sec}$. During this period, and particularly following the injection of the last pellet at $t=5.6$, the fusion power rises sharply. Ignition is indicated in both simulations by the continued increase of the alpha power, $P_{\mathbf{a}}$, following the termination of auxiliary heating. The end of ignition (negative net power into the plasma) occurs at about the time $P_{\alpha}$ peaks. We will discuss the reasons why the discharges did not run away in Sec. III.C. Wall loading considerations require us to limit CIT to a time-averaged alpha power during the burn phase of $P_{\alpha} \simeq 60 \mathrm{MW}$; both of these runs are clearly below this value. The jagged shape of the alpha power curve for simulation II is the result of the sawtooth oscillations. This effect does not appear in I due to the ballooning-enhanced transport which forces the temperature profile to remain relatively $f$ at in the center part of the plasma even between sawteeth in order to stay close to marginal stability with respect to high-n ballooning modes.

The ballooning instability in simulation $I$ is localized inside the sawtooth mixing region ( $\sim 25 \%$ of the minor radius). It is likely that this low-shear, $q \simeq 1$ area is also unstable in II. Note, however, that the volume-averaged toroidal $\beta$ values are well below the Trovon limit ${ }^{38,37}$ of $\beta_{T} \equiv 3 I_{p} / a B_{T} \%$ (where $I_{p}$ is in MA, $a$ in $\mathrm{m}$, and $B_{T}$ in T). The reason for this is that the pressure profiles are not optimized with respect to ballooning modes as are those upon which the Troyon limit is based. For CIT parameters, $\beta_{T}=4.9 \%$. 
In simulation I, we find a peak total $\beta=3.8 \%$ and in $11, \beta=2.7 \%$. These correspond to $3 / \beta_{T}=0.77$ and 0.55 , respectively.

IVe show in Fig. 5 the $\mathcal{B}$ due to fast alpha particles in simulation $I$ as a function of time and plasma half-width. This profile is much more peaked than the thermal pressure profile due to the fact that $P_{a} \sim n_{D} n_{T} T_{i}^{2}$. For this run, the volume-averaged contribution from fast alphas peaks at $\beta_{\alpha}=0.45 \%$; in 11 , we find $\beta_{\alpha}=0.21 \%$.

The evolution of the global energy confinement time, $\tau_{E}$, for the two simulations is illustrated in Fig. 6 . In both cases, pellet injection gives rise to significant changes in confinement. The Singer-Ku transport model typically comes very close to matching neo-Alcator scaling during the ohmic portion of the discharge. This is evidenced in part by the sharp rises in $T_{E}$ at the times of pellet injection ( $\tau_{N_{A}} \propto \bar{n}_{e}$ ). During auxiliary heating, the pellets cause a drop in $T$ that locally decreases thermal transport. With regard to the Redi-Tang model in simulation II, the pellets alter considerably the temperature and density profiles and, hence, lead to changes in the $\nu_{r e}$ and $\eta_{i}$ ralues that are used to choose the thermal diffusivity scaling. Note that in this case the improvement in confirement following pellet injection one expects from the ion channel is not clearly evident. Due to the lack of deep penetration here, the density profile does not peak up sufficiently to raise $\eta_{i}$ above the critical value of 1.5 needed to turn of the $\eta_{i}$ mode transport.

The confinement time in simulation II during the ohmic phase is above the neo-Alrator value. However, the plasma conditions just prior to the initiation of auxiliary heating are not much different from those of simulation $I$, and the plasma energy at this point is small relative to that added by auxiliary and alpha heating. So, we are confident that we are not being overly optimistic in using this transport model during the early stages of the run.

Upon initiation of auxiliary heating, there are again sharp changes in confinement in boih cases. Furthermore, the responses are in opposite directions. In the Tang-Redi transport model, all thermal diffusivities scale with input power and thus show an instantaneous degradation in confinement when the heating is turned on. On the ather hand, because $\chi_{e}$ is proportional to the local temperature in the Singer - Ku model used in simulation I and the heating deposition profile is centrally peaked, we initially see the plasma energy rising rapidly in the center, but remaining relatively fixed near the edge. It is the edge losses that enter into the global $\tau_{E}=W_{\text {lot }} / P_{\text {loss. }}$ Since they do 
not respond to the central heating immediately while the rolume-integrated energy, $W_{\text {to }}$, does, the confinement time actually rises at first. This is true until the centrally deposited energy diffuses out to the edge. Because of the pellet injection at $t=5.6 \mathrm{sec}$, this effect is somewhat obscured in Fig. 6; note, however, that $T_{E}$ is dropping just prior to termination of the auxiliary heating.

In both cases, the degradation (jmprovement) of confinement with increasing (decreasing) alpha power is clear during the burn phase. The oscillations in the curve for sirnulation II are due to the effects of the broad sawteeth. Because of the ballooning-enhanced transport, these oscillations do not appear in simulation $I$.

To get an idea of how the confinement times compare with presently acknowledged scaling laws, let us make a few computations using flat-top parameters. In both cases, $\tau_{N_{A}} \simeq 1.1 \mathrm{sec}$. With $P_{\text {tot }}=60 \mathrm{MW}$ (simulation 1). we have from Eq. $(10), \tau_{E, K G} \simeq 0.45 \mathrm{sec}$. At this point in the run, $\tau_{E}=0.65 \mathrm{sec}$, so $\tau_{E} / \tau_{E, K G} \simeq 1.4$. In this simulation, the ratio $\tau_{E} / \tau_{E, K G}$ is as high as 2.0 during heating; drops steadily during the burn phase, reaching 1.4 near the end of the discharge. The power level is lower in II, $P_{\text {lat }} \simeq 45$ IIV. We calculate $\tau_{E, K G} \simeq 0.52 \mathrm{sec}$ and $\tau_{E} / \tau_{E, K G} \simeq 1.4$ with $\tau_{E} \simeq 0.7 \mathrm{sec}$. For this case, the ratio $\tau_{E} / \tau_{E, K G}$ is close to $1.3-1 . \hat{A}$ throughout the heating and burn phases of the discharge.

Despite the significantly different transport models, and the contrasting profiles that result, these two simulations are qualitatively similar in many respects (for example, see Figs. 3 and 4). This similarity is in part the result of having about the same density and confinement time for both runs.

\section{B. Effects of Sawtooth Osciliations}

As has been noted previously, 9.22 peaked profiles increase the size of the window in density - temperature space for ignited operation. Tisis is primarily due to the fact that $P_{\alpha} \propto n_{D} n_{T} T_{i}^{2}$. That is, with peaked profiles, the central density and temperature are higher for a given volume-averaged energy than with flat profiles and, hence, so is the volume integral of $n_{D} n_{T} T_{i}^{2}$ (i.e., the total alpha power). The time-averaged effect of sawtooth oscillations is to flatten the density and temperature profiles. Hence, they can have a deleterious effect on ignition if they are too broad. 
Bateman ${ }^{22}$ discussed a way to delay significantly the appearance of sawtooth oscillations using a combination of pellet injection and current ramp. Here. we extend this concept and consider factors that might have an impact on the breadth of the sawteeth. In particular, we examine the effects of the thermal transport model and the start-up scenario.

In Figs. $7-10$, we show the ion temperatures and electron densities from our two simulations. The spikes in the electron density during the first six seconds of the discharge are the result of pellet injection. The relatively peaked profile in Fig. 7 is due to the inward particle pinch and the maintenance of relatively narrow sawtooth oscillations; in this case the sawtooth mixing radius is $r_{\operatorname{mix}} / a \simeq 0.25$. In contrast, the profile shown in Fig. 8 is flat during the burn phase even with the same particle pinch; here, $r_{\operatorname{mix}} / a \simeq 0.60$. These sawteeth are large enough to have an impact on the energy confinement time (see Fig. 6). Despite the higher volume-averaged electron density $\left(3.4 \times 10^{20} \mathrm{~m}^{-3}\right.$ versus $\left.3.0 \times 10^{20} \mathrm{~m}^{-3}\right)$, the central density in simulation II is actually smaller. The central density is an important quantity in computing the total alpha power as is demonstrated by the lower fusion output in the second run.

The sawtooth oscillations also affect the ion temperature. Note, however, that thefe are little or no oscillations visible in Fig. 9. This is due not only to the narrow sawteeth, but also to the ballooning-enhanced transport occurring inside the sawtooth mixing region (see Sec. IV.A). The ballooningenhanced transport is not active in case II; this allows the broad sawteeth to combine with rapid heating by the alpha particles to produce large amplitude uscillations appearing during the burn phase; see Fig. 10.

Before proceding any further, we would like to point out that the results of this section are dependent on our choise of wastsoth nodel (see Sec. ii.C). For example, our conclusions would not apply if the actual sawteeth do not flatten the profiles completely inside some surface near $q=1$, but instead produce only a localized flattening. In addition, the length of the sawtooth period can also be questioned since there are no reliable predictions for it. In the case of narrow sawteeth, the magnitude of the period is unimportant. ${ }^{10}$ For broad sawteeth, moderate variations in period should not lead to large changes in the results. ${ }^{9}$

The breadth of the sawtooth oscillations is determined by the shape of the salety factor profile which is related to the current density for the poloidal 
field variable, $B_{p}$, evolved according to Eq. (3)!. Even below ignition temperatures (e.g., a few keV), the resistive skin time for CIT exceeds the length of the discharge. So, the way in which the current is loaded on can affect the plasma behavior during the burn. To demonstrate this, we contrast three different start-up scenarios.

The three schemes differ only in the way we evolve the plasma shape during the ramp phase. First, we will consider a run identical to simulation I but without the bootstrap current (for clarity); this will be referred to as the fast samp case. Secondly, we examine a discharge in which the plasma does not reach full elongation until $t=5.8$ (slow ramp case); as before, the evolution of $\kappa$ and $\delta$ is linear in time. Finally, we carry out a simulation at fixed shape; that is, the plasma starts with an elongation $\kappa=2$.

The reason for ramping the magnetic field (in addition to minimizing resistive heating of the toroidal field coils) and plasma shape is to aid current penetration. Poor current penetration can lead to skin currents and, consequently, to disruptions. ${ }^{1}$ These techniques have an effect even in the absence of resistivity. A brief explanation follows. We have in general $\psi_{t \sigma, a} \simeq B_{T} \pi a^{2} \kappa$, so that raising $B_{T}$ and $\kappa$ leads to an increasing toroidal flux $\psi_{\text {tor.a }}$ within the plasma. As the toroidal field is raised, each surface of constant $\psi_{\text {tor }}$ shrinks in half-width in time. Next, note that

$$
\left.\frac{\partial v_{p \alpha \alpha}}{\partial t}\right|_{\left.\right|_{\psi_{\text {tor }}}}=\frac{2 \pi}{I\left(1 / \boldsymbol{R}^{2}\right\rangle} \eta\left(\langle\boldsymbol{J} \cdot \boldsymbol{B}\rangle-\left\langle J_{\text {beam }} \cdot B\right\rangle-\left\langle J_{\text {boox }} \cdot B\right\rangle\right) .
$$

Taking the derivative of this equation with respect to $\xi$ yields Eq. (3). From Eq. (12), we see that if there is no resistivity $(\eta=0), \psi_{\text {pod }}$ is constant in time on a surface of constant toroidal flux. We also know that at any point in time the toroidal current contained within a flux surface, $I_{p}\left(\psi_{t o r}\right)$, is a flux surface quantity. We can simplify the rest of the argument by referring to circular geometry. In this case, $\psi_{\mathrm{p} \alpha} \propto a R B_{\mathrm{p}}$; by Ampere's law $l_{\mathrm{p}} \propto a B_{\mathrm{p}} \propto \psi_{\mathrm{pod}} / R$. Thus, if $R$ does not change, as is the case here, then $I_{p}$ is (approximately fn general) constant in time for a given value of $\psi_{\text {tor. }}$. In this way we get current penetration due to the shrinking of the constant $\psi_{\text {cor }}$ surfaces alone.

The effects of the magnetic field and shape ramps are additive in this process. So, we expect the fixed shape scheme to yield less current penetration than the schemes with shape ramp. We could apply similar arguments to determine a priori the best shape ramp procedure, but the actual outcome 
also deperids greatly on other factors. For instance, early in the run the current profile is not completely frozen-in. To see this, consider the current density profiles shown in Fig. 11. Before $t \simeq 4 \mathrm{sec}$, the current density peaks up noticeably between sawteeth, as shown in Fig. I1(a). At later times, the profile remains flat throughout the sawtooth period [Fig. 11(b)]. The current density profile is one of the clearest indicators of the breadth of the sawtooth mixing region.

To further elaborate on the differences between the three ramp schemes, consider the time evolution of $q_{\text {cyl }}$ (as defined in Eq. (11)] in Fig. 12. We plot in Fig. 13 the corresponding sawtooth mixing radii as a function of time. Of course, the smaller the sawtooth mixing radius is, the more fusion power we expect due to the profile peaking effect. For $t<4 \mathrm{sec}$, there is a correlation between $r_{m i x}$ and $1 / q_{\text {cyt }}$ in each case. Note that the mixing radius for the fast ramp scheme actually goes through a minimum at about the time $q_{\text {ayd }}$ peaks. In the fixed shape case, the sawteeth disappear between $t \simeq 1.5$ and $t \simeq 8$ sec. During this period, the safety factor on axis, 90 , exceeds unity. Later in the run, $q_{0}$ once again falls below unity, and the sawteeth reappear. These correlations are reminiscent of the observation ${ }^{25}$ that $r(q=1)$;a scales like $1 / q_{e d g e}$, where $r(q=1)$ is the radius of the $q=1$ surface and $q_{a d g e}$ is the edge safety factor.

As the electron temperature, $T_{e}$, begins to rise, $\eta \propto T_{e}^{-3 / 2}$ falls, and the magnetic diffusion slows. This leads to a temporary drop in the rate of change of $r_{\operatorname{mix}}$ for the slow and fast ramp cases.

If we take the derivative of Eq. (12) and use the definition of $B_{p}$ we obtain

$$
\begin{aligned}
\left.\frac{\partial}{\partial t}\right|_{\xi}\left(\rho^{\prime} B_{\mathrm{p}}\right)=\frac{\partial}{\partial \xi} & {\left[\frac{\eta}{I\left\langle 1 / R^{2}\right\rangle R_{0 R E F}}\right.} \\
& \left.\times\left(\langle\boldsymbol{J} \cdot B\rangle-\left\langle J_{\text {beam }} \cdot B\right\rangle-\left\langle J_{\text {boot }} \cdot B\right\rangle\right)\right] .
\end{aligned}
$$

In other words, a higher current density gradient indicates more rapid magnetic diffusion. We see from Fig. 11(b) that the runs with the broader sawtooth oscillations have larger gradients at the edge of the mixing region. Furthermore, these gradients exist in a region of lower $T_{e}$ (larger $\eta$ ) than for, say, the fast shape ramp case. So, while there may be little magnetic diffusion going on with narrow sawteeth (that is, $r_{\operatorname{mix}}$ is not changing much), it is significant, with broad oscillations. This is the trend in Fig. 13. Note 
that the slow ramp case appears to be close to resistive equilibrium at the end of the run.

As expected, there is a correlation between the performance in each of the three cases and the breadth of the sawtooth oscillations. The fast shape ramp scenario evolves similarly to simulation I with a maximum $P_{\alpha}=59 \mathrm{NW}$. The fixed shape run fairs slightly better: the peak $\boldsymbol{P}_{\alpha}=63 \mathrm{MW}$. Furthermore, the burn lasts about a half second longer. Not surprisingly, the slow ramp case is the worst with $P_{\alpha}$ getting no higher than $22 \mathrm{MW}$; there is only a brief period following auxiliary heating during which the plasma is ignited.

Although the fixed shape case may be the most desirable as far as fusion power output is concerned, it is not from a stability standpoint. The current profile in Fig. $11(\mathrm{~b})$ is clearly non-monotonic, indicative of skin currents. A check of the internal inductance values, $\ell_{i}$, from this run indicates that it is likely to be unstable with respect to the internal kink mode. ${ }^{39}$ The fast ramp case might also be susceptible to such instabilities, but a more precise stability calculation will be required to make this determination.

In general, it appears that the optimum ramp scheme would be such that $q_{c y l}$ drops monotonically from the initial value of the shape-ramped cases to the final, flat-top state. This should provide enough current penetration to avoid skin currents, yet not drop so low in $q$ as to yield broad sawtooth oscillations or to cause disruptions.

The ramp scheme may not be important if the electron temperature profiles resemble the ones obtained in simulation II. In simulation I, the SingerKu thermal transport model yields an electron thermal diffusivity, $\chi_{e}$, that rises slowly from $\sim 0.2 \mathrm{~m}^{2} / \mathrm{sec}$ near the center to $\sim 0.4 \mathrm{~m}^{2} / \mathrm{sec}$ at the edge. In contrast, the Redi-Tang model leads to considerably larger edge values, $\chi_{e}>1-2 \mathrm{~m}^{2} / \mathrm{sec}$, while the central diffusivity is also on the order of 0.2 $\mathrm{m}^{2} / \mathrm{sec}$, or smaller. Most of the increase with radius takes place in the outer $20 \%$ of the plasma. The effects of this can be seen directly on the edge ion temperature profiles in Fig. $10\left(\chi_{i}\right.$ exhibits the same characteristics so that $T_{e}$ and $T_{i}$ have similar shapes); this is essentially the Gaussian profile shape assumed in the Redi-Tang model. On the other hand, in the Singer-Ku transport model, $T_{i}$ drops almost linearly with increasing radius.

During the flat-top phase of the discharge, $V_{\text {loop }}=\partial \psi_{\text {pol }} / \partial t$ is approximately constant in space near the edge. Hence, we have $J_{\text {cor }} \propto \eta^{-1} \propto T_{e}^{3 / 2}$, neglecting neoclassical resistivity effects. Indeed, the current density profiles 
in Fig. 11 look similar to the ion temperature profile, even at $t=3 \mathrm{sec}$. Because of the large volume elements at the edge relative to the center, there is little current in the outer $25-30 \%$ of the plasma in simulation II compared to I. Since both cases utilize the same total current, case II must have more current near the magnetic axis. Again, this is apparent in Fig. 11. As a resuit, we compute a broader safety factor profile and greater sawtooth mixing radius in this run. The low value of $J_{\text {tor }}$ near the edge accompanying the Redi-Tang transport model also implies a gradient that is even larger than the one noted in the slow ramp case. Hence, the magnetic diffusion is rapid, and the rate of increase of $r_{\text {mix }}$ with time is high. Early in the current ramp, this effect is not so important, and $r_{\text {mix }}$ evolves as in the fast ramp case (both utilize the same start-up scheme). Resistive equilibrium is reached at $t \simeq 8$ sec.

We have used the Redi-Tang model previously in simulating CIT. One of the studies attempted to mock up $\mathrm{H}$-mode behavior by artificially decreasing the electron and ion thermal diffusivities near the edge. ${ }^{33}$ In these runs, not only did we find that the energy confinement improved significantly, but also that the sawtooth mixing radii were much smaller than expected. The narrower sawteeth were a result of the broader current profiles accompanying the high edge temperatures predicted by this transport model. It is reasonable to conclude that this behavior substantiates the above arguments.

\section{Effects Leading to Ignition Termination}

From Fig. 3 it is clear that the ignition in simulation $l$ ends at $t \simeq 9$ sec. This is a result of the drop in the density of deuterium and tritium ions during the burn phase (recall that the electron density is being held constant). These losses result from a combination of helium ash accumulation and displacement by influxed carbon atoms. Since the code does not allow either of these impurity species to diffuse out, both effects lead to a hydrogenic ion density that is monotonically decreasing in time. In particular, the total drop in the volume-averaged hydrogenic jon density between $t=6.5$ and $t=10.8$ is $\Delta\left\langle n_{D}+n_{T}\right\rangle=-5.4 \times 10^{19} \mathrm{~m}^{-3} ;$ at $t=6.5,\left\langle n_{D}+n_{T}\right\rangle=2.6 \times 10^{20} \mathrm{~m}^{-3}$. Of this change, helium accumulation accounts for $79 \%$; displacement by carbon accounts for the other $21 \%$.

An often-used measure of the performance of a particular ignition sce- 
nario is the product $\left\langle n_{D}+n_{T}\right\rangle \tau_{E}\left\langle T_{i}\right\rangle$, where $\tau_{E}$ is the global energy confinement time and $\left(T_{i}\right)$ is the density-weighted, volume-averaged ion temperature. This parameter characterizes the balance between alpha power $\left(\propto\left\langle n_{D} n_{T} T_{i}^{2}\right)\right)$ and the total losses $\left(\propto\left(n_{D}+n_{T}\right)\left\langle T_{i}\right\rangle / \tau_{E}\right.$, given that we can relate the hydrogenic ion energy to the total plasma energy by a constant factor). In simulation the Singer-Ku model simulation (I), we find that between $t=6.5$ and $t=9.0$ (when $P_{\text {net, }}$, the time rate of change of the total plasma energy, first becomes negative), $\tau_{E}\left\langle T_{i}\right\rangle$ actually increases. However, this is more than offset by the decrease in hydrogenic ion density over the same time interval so that $\left\langle n_{D}+n_{T}\right\rangle \tau_{E}\left\langle T_{i}\right\rangle$ falls below the critical value for ignition.

The loss of deuterium and tritium also seems to be instrumental in ending the ignition in the Tang model simulation (II). In this case, $\Delta\left\langle n_{D}+n_{T}\right\rangle=$ $-4.2 \times 10^{19} \mathrm{~m}^{-3}$ between $t=6.5$ and $t=10.8$, with $\left\langle n_{D}+n_{T}\right\rangle=2.9 \times 10^{20}$ $\mathrm{m}^{-3}$ at $t=6.5$. Helium ash buildup is responsible for $72 \%$ of the change; displacement by carbon accounts for the rest. The effects are snaller (the ignition ends more slowiy) in this run due to the lower $P_{\alpha}$ and higher $\left\langle n_{e}\right\rangle$. Other effects that might be contributing to the termination of ignition in this case, and in our CIT simulations in general, will be described below.

We will now describe the conditions under which we expect the burnup of deuterium and tritium to be significant and then demonstrate what happens if the helium ash can be removed. This discussion can be better understood if we speak in terms of a O-D power balance calculation (Plasma OPeration CONtours or POPCON plots, see Refs. 8,9,38, and 40). We display the results of these computations as contours of constant $P_{A U X}$, the auxiliary power required to maintain steady state, in $\left\langle n_{e}\right\rangle$ vs. $\left\langle T_{e}\right\rangle$ space. For clarity, we show in Fig. 14 a "typical" POPCON plot for CIT using a $\tau_{E}$ scaling comparable to that obtained in the present simulations. More precise POP. CONs for CIT have been given elsewhere. ${ }^{36}$ Note that we can also loosely interpret $P_{A U X}$ as the negative of the time rate of change of the total plasma energy (i.e., $-P_{\text {net }}$ ) in the absence of auxiliary power input. The $P_{A U X}=0$ contour on the left side of the diagram represents ohmic equilibrium, while the one in the upper right corner denotes ignited equilibrium.

This POPCON plot is the result of solving

$$
P_{\alpha}+P_{O H}+P_{A U X}=\frac{\frac{3}{2} \sum_{j} n_{j} T_{j}}{\tau_{E}}+P_{R A D}
$$


using a 0-D code, ${ }^{9.38,40}$ where $P_{\alpha}$ is the alpha power computed using prescribed profiles (parabolas with a user-specified exponent), $P_{O H}$ is the ohmic heating power, $P_{R A D}$ is the power loss due to bremsstrahlung radiation, the sum over $j$ includes ions and electrons, and $\tau_{E}$ is calculated using some userspecified formula, for example Eq. (10). The equilibrium ignition contour goes to higher densities at the highest temperatures in part as a result of the effective exponent on $T_{i}$ in the $P_{\alpha}$ formula falling below 2 when $T_{i}>20 \mathrm{keV}$.

When the electron density is held constant with no ash removal, ignited steady states at low density, but high temperature (so that the alpha power is significant) will be the most susceptible to deuterium - tritium burn up. In the POPCON plot, these conditions are represented by the lower right-hand portion of the ignited equilibrium contour.

Conversely, discharges with high density and low temperature (upper left of the ignited $P_{A U X}=0$ contour) will be the least affected by helium ash accumulation. This is due not only to the higher density itself, but also to the lower $F_{\alpha}$ under these conditions. Furthermore, the effect of a given drop in density on $P_{A U X}$ is smaller at very large densities. That is, the ignition contour in this case is given by the balance between $P_{\alpha}$ and $P_{\text {RAD }} \propto n_{\varepsilon}^{2} T_{\varepsilon}^{1 / 2}$ so that there is no density dependence to the $P_{A U X}=0$ contour.

We should point out that the left side of the ignited equilibrium contour is thermally unstable, $d P_{A U X} / d\left\langle T_{e}\right\rangle<0 .^{8}$ That is, a perturbation to the right will lead to a growing plasma energy; the resulting higher temperature raises $-P_{A U X}$ even more. This process would continue (if constant density were maintained) until $d P_{A U X} / d\left(T_{e}\right)$ changes sign (towards the right side of the ignited equilibrium contour). As a result, an ignited plasma with high density and low ten perature would have to be held very close to the $P_{A U X}=0$ contour to prevent such a runaway from creating higher $P_{\alpha}$ and significant deuterium - tritium burnup.

We now return to simulation I and consider the effects of removing the helium ash as it is formed. This is done by specifying a negative impurity influx. To get effective pumping we find it necessary to flatten the helium density profile by altering its diffusion model. Although this is not an experimentally realistic procedure, it does clearly show the impact the helium ash can have on CIT performance. The results of this simulation are depicted as the second $P_{\alpha}$ curve in Fig. 3. Note that the alpha power keeps increasing after ignition has ended in simulation I and appears to approach a steady 
state near $t=10.8 \mathrm{sec}$. The total loss of deuterium and tritium between $t=6 . \overline{5}$ and $t=10.8$ is $\lambda\left\langle n_{D}+n_{T}\right\rangle=-1.1 \times 10^{19} \mathrm{~m}^{-3} ; 91 \%$ of this is due to displacement by the influxed carbon atoms. In fact, $\left(n_{D}+n_{T}\right)$ is very steady for $t>8 \mathrm{sec}$.

It is not completely clear why $P_{\mathbf{a}}$ does not continue to increase past 70 MW. If we again consider the product $\left\langle n_{D}+n_{T}\right\rangle \tau_{E}\left\langle T_{i}\right\rangle$, we find that now with $\left\langle n_{D}+n_{T}\right\rangle$ approximately constant and $\left\langle T_{i}\right\rangle$ increasing up to $t=9 \mathrm{sec}$, it is a drop in $\tau_{E}$ that causes the plasma conditions to stabilize. In particular, the conducted power seems to be increasing faster than $(T)^{2}$. On the other hand, the central temperatures approach $30 \mathrm{keV}$ in this run. At that level, the drop in $P_{\mathrm{a}}$ relative to $\left\langle n_{D} \pi_{\mathrm{T}} T_{i}^{2}\right\rangle$ could be important.

We can best explain the evolution of this simulation in terms of a POP. CON diagram. The racio $\left\langle n_{D}+n_{T}\right\rangle /\left\langle n_{e}\right\rangle$ is taken to be a constant over the entire $\left\langle n_{e}\right\rangle$ vs. $\left\langle T_{e}\right\rangle$ space. Varving the parameter representing this ratio leads to a family of $P_{A U X}=0$ contours. If we instead imagine plotting the contours with an ordinate of $\left\langle n_{D}+n_{T}\right\rangle_{,}$we can depict the trajectory of a discharge with time-varying deuterium and tritium density.

In the simulation with helium pumping, we find that after the auxiliary heating is turned off, $P_{\text {net }}$ (i.e., $-P_{A U X}$ ) first increases with temperature and then falls. Near the end of the run, the plasma density, temperature, etc. approach steady values with $P_{\text {ret }}<1 \mathrm{MW}$. On the POPCON plot, this means a trajectory starting (when the ICRF heating is completed) on the left side of the ignition region. It moves to the right with increasing temperature (going down slightly as the density drops a bit); in this way $-P_{\text {AUx }}$ rises, then falls. Finally, a stable steady state would be reached on the right side of the $P_{A U X}=0$ contour (see Fig. 15).

So, it appears that the ignition contours predicted by BALDUR have the same shape as the ones in Fig. 14. However, the detailed values of $P_{A U X}$ (or $P_{\text {net }}$ ) for a given density and temperature may not be the same (e.g., the 0-D code is not capable of modelling subtle transport effects). Even if the steady state is reached as a result of some transport-model-dependent feature, the details of the fusion cross section that give rise to the shape of the iznition contour in the POPCON diagram are real effects and likely would have led to a similar conclusion to the run (for the $\tau_{E}$ scaling used here).

In simulation I, the trajectory in $\left\langle n_{D}+n_{T}\right\rangle-\left\langle T_{e}\right\rangle$ space begins at the same point as the heljum-pumped run following ICRF heating. The trajectory 
again moves to the right, but it also moves down as the fuel is consumad. It continues in this way until the $P_{A U X}=0$ contour is reached. Since $P_{A U X}$ generally increases with decreasing density, there is no steady state here (fuel consumption continues). Hence, $P_{A U X}$ becomes positive as the run continues so that the plasma loses energy, and the temperature drops until the discharge is terminated (see Fig. 15).

All of the effects described in this subsection are important in our simulations because the plasma conditions are so close to the bottom of the ignited $P_{A U X}=0$ contour in the POPCON diagram of Fig. 15. If the plasma density were much higher or the mergy confinement time scaling much more favorable, this would not is the case. Then, we would see an appareut thermal runaway for the (short) duration of a CIT discharge. We ran also conclude here that control of the plasma density is one suitable method of burn control. That is, if the density rises uncontrollably, we might end up with a real runaway discharge.

We now mention briefly other effects that have had an impact on the burn phase of these and previous simulations. Perhaps most important is the broadening of the sawtooth mixing radius with time (see Fig. 13). The resulting flattening of the profiles leads to a decreasing alpha power even if the deuterium - tritium density is maintained. These effects are even greater if ballooning-enhanced transport is included.

Two subtler phenomena could affect a very marginal discharge. The first of these involves the ragiation increase that follows the inward diffusion of the carbon influxed during the burn phase. Since most of the radiation in these simulations is due to bremsstrahlung, this rise is just a result of a higher $Z_{e f f}$ in the hot plasma core. Finally, in cases with large amounts of helium pumping, the deuterium - tritium gas puffing required to maintain constant electron density can be signifcant. This leads to a higher neutral density near the edge and, consequently, to increased charge exchange losses.

\section{Sensitivity to Assumptions}

In this section we will discuss briefly how our results depend on: (i) whether or not the profiles are constrained to be nearly stable with respect to high- $n$ ballooning modes, (ii) the shape of the heating profile, (iii) inclusion of the bootstrap current, and (iv) the particle transport model. This list of 
topics is intended to complement the sensitivity tests performed in Ref. 10 .

\section{A. Ballooning Modes}

Simulation I as described above includes the ballooning-enhanced transport. ${ }^{9}$ Removing the enhancement leads to very little difference in the results. The profiles do not show much effect due to the relatively small sawtooth mixing radius. The peak values become $\beta=4.5 \%$ and $P_{a}=71 \mathrm{MW}$; both are slightly higher than in the original run $\left(\beta=3.8 \%, P_{\alpha}=60 \mathrm{MW}\right)$. Furthermore, the ignition phase develops and ends in the same manner as discussed in Sec. III.A. The only quantities exhibiting any significant change are the central temperatures; without the ballooning-enhanced transport, they can peak up at the end of each sawtooth rise (e.g., as in Fig. 10).

As noted previously, simulation II does not include the ballooning-enhanced transport. Due to the broad sawtooth oscillations, enforcing ballooning stability has a great effect on the outcome of the calculation. When this is done, the central temperatures remain essentially flat out to the mixing radius thro ighout each sawtooth rise. The resulting decrease in fusion power leads to much poorer performance. The simulation with ballooning mode-enhanced transport does ignite, but only for a fraction of a second. We find peak parameters yell below those of simulation II: $\beta=1.8 \%$ and $P_{a}=22 \mathrm{MW}$.

From these runs, we conclude that the consequences of enforcing ballooningstable profiles depend greatly on the size of the sawtooth mixing radius. Furthermore, due to the fact that the modes are typically unstable only inside of the mixing radius (with a critical pressure gradient of almost zero), the effects of the ballooning-enhanced transport are almost equivalent to greatly reducing the sawtooth period.

Many of the assumptions made in deriving the ideal MHD ballooning mode equation ${ }^{9}$ do not strictly hold in an ignited CIT. In particular, the large, central, low-shear region and nonthermal alpha particles are not allowed for in the orderings leading to it. The appropriate calculations are far too complicated to be included as part of the BALDUR simulations. Insiead, we transfer information about the equilibrium profiles to other groups to be analyzed in a more rigorous fashion.

The PEST stability code has been used to analyze the data from simulation $\mathrm{L}^{+1}$ In addition to looking at high-n ballooning and Mercier modes, this 
code considers low-n, pressure-driven modes in the low shear limit (infernal modes ${ }^{12}$ ) and external kinks. The low shear region inside of the sawtooth mixing radius in simulation I is predicted to be unstable with respect to Mercier and other pressure-driven modes, but is otherwise stable. Instability is predicted in the center despite the activity of the ballooning-enhanced thermal transport in part because of the absence of ballooning-enhanced particle transport in our model. The peaking of the density profile during the sawtooth rise due to the inward pinch leads to a small, but non-zero pressure gradient.

Rewoldt has investigated the effects of the high-energy alpha particles on high-n instabilities. ${ }^{43}$ The data used were taken at $t=9$ sec from a preliminary run qualitatively similar to simulation I. The calculation was performed on a flux surface near $r / a=0.5$. He found that the alpha particles have a weak effect on the trapped electron drift mode, but substantially lowered the critical $\beta$ for the kinetically calculated MHD ballooning mode. In fact, this ballooning mode was predicted to be unstable for the parameters used. The thermalized helium ash does little to alter the behavior of either instability.

\section{B. Heating Profile}

The width (and location of the peak) of the ICRF deposition profile can affect the energy confinement time. As was discussed in Sec. III.A, $\tau_{E}$ actually rises in simulation I with the onset of auxiliary heating; this is in part the result of the narrow deposition profile used. We have tried broader heating profiles not only to verify that this explanation is reasonable but also to determine how important these effects are to CIT performance. Thus far, we have only looked at simulations involving the Singer-Ku transport model.

We find that if the heating profile is broad enough (for example, dropping to half of its central value by $r=0.4 \mathrm{~m}$ ), the confinement time does fall when the auxiliary heating is turned on and remains lower than in the case with a narrow profile (ignoring the effects of pellets). Depending on how peaked the heating is, CIT may require more time or auxiliary power to reach ignition temperatures. Both simulations I and II show $P_{\text {net }}>0$ when the auxiliary power is turned off. That is, they would actually ignite at lower temperatures. So, there is some room for a broader profile even within the present scenarios. 
Previous simulations of CIT using the BALDUR code ${ }^{10}$ have shown that the location of the peak affects performance; a centrally peaked profile is most desirable. In that a profile peaking off-axis deposits more power closer to the edge, their results are consistent with our conclusions regarding the width of the heating profile. We would aiso like to point out that there is now some evidence in experimental data for the shapes of heating profiles affecting the energy confinement time. ${ }^{44}$

The alpha-heating model used in the above simulations does not allow sawtooth mixing of the high energy alpha particles. It is not certain from a theoretical standpoint whether or not this mixing should occur in an actual experiment. In order to determine if it matters, we now describe the results of a simulation using a simpler alpha-heating mode $\left.\right|^{11}$ that does allow mixing of the slowing-down particles. The run used for the comparisun is simulation II; the broad sawtooth oscillations should nearly maximize the effect. However, we find that the various temperatures and $\beta$ values vary between the two runs by only a few percent or less; the peak alpha power is $P_{\alpha}=43 \mathrm{MW}$, much as it is in simulation II. Furthermore, the details of the evolution of the burn phase are virtually unaltered.

We might have expected some effect from this procedure due to the alterations in the effective alpha-heating profile. The reason we do not may have to do with the fact that all of the changes are taking place inside of the sawtooth mixing radius. The confinement of the bulk plasma energy within this region is largely governed by the sawtooth oscillations, so that varying the heating profile here produces only negligible effects.

\section{Bootstrap Current}

We ccnsider two runs: one with the bootstrap current and one without. Simulation I serves as the former, and the fast ramp case used in the dis-

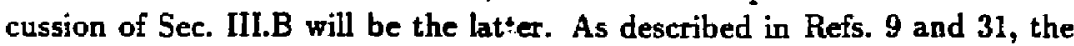
bootstrap current, $J_{\text {boot }}$, is proportional to a sum of density and temperature gradients and vanishes at the magnetic axis. Hence, $J_{\text {boot }}$ peaks off-axis. In simulation I, the peak occurs just outside of the sawtooth mixing radius; the bootstrap current accounts for approximately $20 \%$ of the total current at this radius during the burn phase. With the profiles in use here, $J_{\text {boot }}$ falls more slowly with $r$ than the total current density; it is never so large that the 
loop voltage becomes negative. Thus, it is not too surprising that we find the total cursent profile in the case without bootstrap current to be slightly more peaked than in simulation. This gives rise to the approxinately $0.04 \mathrm{~m}$ increase in the mixing radius from the value quoted in Sec. III.B $(\sim 0.14 \mathrm{~m})$ to that shown in Fig. 13. We also note that the bootstrap current tends to smooth out the current profile; there is otherwise a ledge just outside of the mixing radius [see Fig. 11(b)].

Although the bootstrap current appears to be of limited importance in CIT, it need not be in other reactor designs. There may be more interest in this neoclassical effect since there is now experimental evidence of its existence. ${ }^{3}$

\section{Particle Transport Model}

The main impact of the particle transport model in CIT is on the density profile during the burn phase. In particular, the shape of the density profile is determined by the ratio $v / D$ [see $E q$. (6)]. This effect enters through the alpha power which increases roughly with the square of the central density (see Sec. IIt.B). So in general, the fusion power can be altered either by changing the volume-averaged density (Fig. 14) or by forcing the density profile to have a different shape with the same volume average. ${ }^{22}$ If it turns out that the density profiles in CIT are flat (as they are in a typical H-mode), the performance might be degraded considerably from what is described in this paper. This concern motivated in part the inclusion here of simulation II. Its broad sawteeth lead to a flat density profile despite the presence of the inward pinch; the effects of the relatively low central density are apparent in Fig. 4. In general, a flat density profile requires a higher volume-averaged density or better energy confinement to achieve the same performance l:svel as a discharge with a peaked profile. If CIT does exhibit flat density profiles, it will likely need to be operated at or above the most conservative estimates of the density limit (see Sec. II.A) in order to reach ignition.

\section{Conclusions}

In this paper, we have presented detailed 1-1/2-D transport simulations of CIT that include time variations of fields, densities and plasma shape, 
as well as various MHD and neoclassical effects. Two thermal transport models are employed; both are constrained to yield a scaling fot the global energy confinement time that is better than L-mode [as defined by Eq. (10)]. The temperature profiles from the two simulations differ significantly due to the dissimilar sawtooth oscillation widths. The responses in $\tau_{E}$ in each case to transients such as pellet injection and the initiation of auxiliary heating are not the same. Yet, both simulations result in an ignition period of at least two seconds. The run with the broader sawtooth oscillations requires a somewhat higher density to allow ignition to be achieved.

From these simulations, previous unpublished simulations and various 0 D calculations, we conclude that CIT should ignite if a confinement scaling of $\tau_{E} / \tau_{E . K G} \simeq 1.4 \mathrm{can}$ be maintained at power levels up to $60 \mathrm{MW}$.

That broad sawtooth oscillations can hinder reactor performance has been suggested previously. ${ }^{9,22}$ The long resistive skin time in CIT compared with the length of the discharge means that the sawtooth mixing radius during the burn phase depends on how the current is loaded during the start-up. We contrasted three different start-up scenarios (Sec. III.B) using the SingerKu transport model ${ }^{23}$ to demonstrate this point. In particular, it appears that in CIT avoiding skin currents requires both magnetic field and plasma shape ramps. At the same time, preventing broad sawtooth oscillations (and disruptions) constrains $q_{\mathrm{es}}$ from dropping too low during this period. The optimum scheme may be one in which $q_{c y}$ falls monotonically in time from its initial value with a circular cross-sectional plasma to its final state with an elongated cross section.

After studying the burn phases of the discharges presented here, we conclude that if the volume-averaged electron density is held fixed and helium ash not removed, ignition ends due to the drop in deuterium - tritium density. This is demonstrated in Fig. 3 where we show a simulation in which helium is pumped as it is formed; in this case, a nearly steady-state ignition is achieved by the end of the run. The alpha power remains reasonable because CIT does not have a great deal of ignition margin at these parameters.

It is clear from this work that control of the deuterium and tritium densities is equivalent to burn control. On the one hand, if the fuel densities cannot be maintained, ignition will cease. On the other, if the plasma density cannot be restrained, the plasma will likely run away.

The primary concern of future work should be the development of more re- 
liable transport models and scaling laws; this is also the conclusion of Singer, et al. in Ref. 10. Although we have relied here on the Kaye-Goldston scaling law for our reference L-mode energy confinement t:me $\left(\mathrm{Eq}_{\mathrm{q}}(10)\right.$ ), there are others that are not nearly as favorable for CIT. ${ }^{25,45}$ As was noted above, CIT will need to do even better than Kaye-Goldston L-mode in order to ignite. So, schemes for enhanced confinement operation are also needed. Transport models for the H-mode are currently being developed. ${ }^{48}$ Newer ways of enhancing confinement have been recently discovered ${ }^{3,47}$; their applicability to CIT is uncertain, however.

The above-stated requirements on the energy confinement time can be relaxed somewhat if the electron density is allowed to exceed the values used here (see Sec. II.A). Fortunately, there is now some evidence, given by Greenwald, that this is reasonable. ${ }^{19}$ On the other hand, more work is needed to widen the experimental data base for the Greenwald scaling and to provide theoretical explanations and transport models (see, for example, Ref. 48). Even if densities higher than $3 \times 10^{20} \mathrm{~m}^{-3}$ are possible, there is some concern with the fuelling of the device at that level. ${ }^{19}$ It is likely that a reliable pellet injector capable of significant penetration (during at least the low temperature phase of the discharge) will be required.

\section{Acknowledgement}

This work was supported by U.S. DOE Contract No. DE-AC02-76-CHO3073. 


\section{References}

'D. POST, W. HOULBERG, G. BATEMAN et al., "Physics Aspects of the Compact Ignition Tokamak," Physica Scripta T16, 89 (1987).

2J. LUXON, P. ANDERSON, F. BATTY, C. BAXI, G. BRAMSON, et al., "Initial Results for the DIIJ-D Tokamak," Proc. 11th Int. Conf. Plosma Physics and Controlled Nuclear Fusion, Kyoto, Japan, November 13-20, 1986, p. 159, IAEA-CN-47/A-III-3, International Atomic Energy Agency.

${ }^{3}$ R. J. HAWRYLUK, V. ARUNASALAM, M. G. BELL, M. BITTER, W. R. BLANCHARD, et al., "TFTR Plasma Regimes," Proc. 1lth Int. Conf. Plasma Physics and Controlled Nuclear Fusion, Kyoto, Japan, November 13-20, 1986, p. 51, IAEA-CN-47/A-I-3, International Atomic Energy Agency.

"JET Team: H. ALTMANN, R. J. ANDERSON, J. ARBEZ, W. BAILEY, D. V. BARTLETT, et al., "JET Latest Results and Future Prospects," Proc. 11th Int. Conf. Plasma Physics and Controlled Nuclear Fusion, Kyoto, Japan, November 13-20, 1986, p. 31, IAEA-CN-47/A-I-2, International Atomic Energy Agency.

5.H. H. REDI, W. M. TANG, P. C. EFTHIMION, D. R. MIKKELSEN, and G. L. SCHMHDT, "Transport Simulations of Ohmic TFTR Experiments with Microinstability Based Profile Consistent Models for Electron and Ion Thermal Transport," Nucl. Fusion 27, 2001 (1987).

${ }^{6}$ R. R. DOMINGUEZ and R. E. WALTZ, "Tokamak Transport Code Simulations with Drift Wave Models," Nucl. Fusion 27, 65 (1987).

${ }^{7}$ R. J. GOLDSTON, "Topics in Confinement Analysis of Tokamaks with Auxiliary Heating," Proc. Course and Workshop Basic Physical Processes of Toroidal Fusion Plasmas, Varenna, Italy, August 26 - September 3, 1985 , p. 165, EUR-10418-EN, CEC, Brussels.

${ }^{8}$ W. A. HOULBERG, S. E. ATTENBERGER, and L. M. HIVELY, "Contour Analysis of Fusion Reactor Plasma Performance," Nucl. Fusion 22, 935 (1982). 
${ }^{9}$ D. P. STOTLER. D. POST, and G. BATEMAN, "Sawtooth Effects in INTOK and TIBER." PPPL-2463, Princeton Plasma Physics Laboratory (1987). (submitted for publication in Fusion Technology).

${ }^{10} \mathrm{C}$. E. SINGER, G. BATEMAN, and L. P. KU, "Plasma Transport in a Cumpact Ignition Experiment," PPPL-2414, Princeton Plasma Physics Laboratory (1987) (submitted for publication in Fusion Technology).

${ }^{11}$ C. SINGER, D. POST, D. MIKKELSEN, M. REDI, A. MCKENNEY, A. SILVERMAN, F. SEIDL, P. RUTHERFORD, R. HAWRYLUK, W. LA.VGER, L. FOOTE, D. HEIFETZ, W. HOULBERG, M. HUGHES, R. JE.VSEN, G. LISTER, and J. OGDEN, "BALDUR: A One-Dirnensional Plasma Transport Code," PPPL-2073, Princeton Plasma Physics Laboratory (1986) (to appear in Computer Physics Communications).

${ }^{12}$ G. BATEMAN, "Algorithms for 1-1/2-D Transport," in Praceedings of the 1985 Trieste Spring College on Plasma Physics (to be published).

${ }^{13} \mathrm{~F}$. WAGNER, G. BECKER, K. BEHRINGER, D. CAMPBELL, A. EBERHAGEN, et al., "Regime of Improved Confinement and High Beta in Neutral-Beam-Heated Divertor Discharges of the ASDEX Tokamak," Phys. Rev. Lett. 49, 1408 (1982).

${ }^{14}$ J. C. DeBOO, "Confinement Studies in DIII-D," Bull. Am. Phys. Soc. 32, $1711(1987)$.

${ }^{15}$ S. M. KAYE and R. J. GOLDSTON, "Global Energy Confinement Scaling for Neutrai-Beam-Heated Tokamaks," Nucl. Fusion 25, 65 (1985).

${ }^{16} \mathrm{~N}$. POMPHREY, S. C. JARDIN, and J. MANICKAM, "One Complete Discharge in the Compact Ignition Tokamak," Bull. Am. Phys. Soc. 32, 1982 (1987).

${ }^{17}$ M. AIURAKAMI, J. D. CALLEN, and L. A. BERRY, "Some Observations on Maximum Densities in Tokamak Experiments," Nucl. Fusion 16, 347 (1976).

${ }^{18}$ K. B. AXON, W. H. M. CLARK, J. G. CORDEY, M. COX, S. J. FIELDING, et al., "Beam-Driven Currents, Power Balances and Density Limits with Neutral Injection into DITE," Proc. 8th Int. Conf. Plasma Physics 
und Controlled Nuclear Fusion, Brussels, Belgium, July 1-10, 1980, p. 413. IAEA-CN-38/N-4, International Atomic Energy Agency.

${ }^{19}$ M. GREENWALD, M. BESEN, F. CAMACHO, C. FIORE, M. FOORD, et al., "Studies of the Regime of Improved Particle and Energy Confinement following Pellet Injection into Alcator C," Proc. 11th Int. Conf. Plasma Physics and Controlled Nuclear Fusion, Kyoto, Japan, November 13-20, 1986. p. 139, 1AEA-CN-47/A-111-1, International Atomic Energy Agency.

${ }^{20} \mathrm{~F}$. H. SEGUIN, R. PETRASSO, and E. S. MARMAR, "Effects of Internal Disruptions on Impurity Transport in Tokamaks," Phys. Rev. Lett. 51, $45 \overline{5}$ (1983).

${ }^{21}$ M. GREENWALD, D. GWINN, S. MLORA, J. PARKER, R. PARKER, et al., "Pellet Fuelling Experiments in Alcator-C," Proc. 10th Int. Conf. Plasma Physics and Controlled Nuclear Fusion, London, U.K., September 12-19, 1984, p. 45, IAEA-CN-44/A-I-3, International Atomic Energy Agency.

${ }^{22}$ G. BATEMAN, "Delaying Sawtooth Oscillations in the Compact Ignition Tokamak," PPPL-2373, Princeton Plasma Physics Laboratory (1986) (to appear in Fusion Technology).

${ }^{23}$ C. SINGER. L.-P. KU, G. BATEMAN, F. SEIDL, and M. SUGIHARA, "Physics of Compact Ignition Tokamak Designs," Proc. 11th Symp. Fusion Engineering, Austin, Texas, November 18-22, 1985, p. 41.

${ }^{27} \mathrm{C}$. S. CHANG and F. L. HINTON, "Effect of Finite Aspect Ratio on the Veoclassical Ion Thermal Conductivity in the Banana Regime," Phys. Fluids 25, 1493 (1982).

${ }^{25}$ R. J. GOLDSTON, "Energy Confinement Scaling in Tokamaks: Some lmplications of Recent Experiments with Ohmic and Strong Auxiliary Heating," Plasma Phys. Controlled Fusion 26, 87 (1984).

${ }^{26}$ M. KEILHACKER, G. BECKER, K. BERNHARDI, A. EBERHAGEN, II. EISHAER, et al., "Confinement Studies in L and H-Type ASDEX Discharges," Plasma Phys. Controlled Fusion 26, 49 (1984). 
${ }^{27} \mathrm{X}$. A. UCKA.N, W. A. HOULBERG, and J. SHEFFIELD, "Physics Evaluation of Compact Tokamak Ignition Experiments, ${ }^{7}$ Proceedings 11th Symp. Fusion Engineering, Austin, Texas, November 18-22, 1985, p. 401.

${ }^{28}$ B. B. KADOMTSEV, "Disruptive Instabilities in Tokamaks," Sov. $J$. Plasma Phys. 1, 389 (1975).

${ }^{29} \mathrm{~J}$. W. CONNOR, J. B. TAYLOR, and M. F. TURNER, "Ideal MHD Ballooning Instability and Scaling Law for Confinement," Nucl. Fusion 24, 642 (1984).

${ }^{30}$ M. AZUMI, T. TSUMENATSU, K. ITOH, T. TUDA, G. KURITA, et al. "Evolution of Stable High-Beta Tokamak Equilibria," Proc. 8th Int. Conf. Plasma Physics and Controlled Nuclear Fusion, Brussels, Belgium, July 1-10, 1980, p. 293, IAEA-CN-38/K-1-1, International Atomic Energy Agency.

${ }^{31}$ S. P. HIRSHMAN and D. J. SIGMAR, "Neoclassical Transport of Impurjties in Tokamak Plasmas," Nucl. Fusion 21, 1079 (1981).

${ }^{32}$ S. P. HIRSHMAN, "Neoclassical Current in a Toroidally-Confined Multispecies Plasma," Phys. Fluids 21, 1295 (1978).

${ }^{33}$ D. P. STOTLER, G. BATEMAN, and M. H. REDI, "Transport Simulations of the Compact Igrition Tokamak," Sherwood Theory Meeting, San Diego, California, 1987, paper 2D28.

${ }^{34}$ P. COLESTOCK, Princeton Plasma Physics Laboratory, private communication.

${ }^{35} \mathrm{C}$. K. PHILLIPS, Princeton Plasma Physics Laboratory, private communication.

${ }^{36}$ A. SYKES, M. F. TURNER, and S. PATEL, "Beta Limits in Tokamaks Due to High-n Ballooning Modes," Proc. 11th European Conf. Controlled Fusion and Plasma Physics, Aachen, FRG, September 5-9, 1983, p. 363, European Physical Society. in Controlled Fusion and Plasma Physics, (11th European Conference, Aachen, European Physical Society), Vol. 7D, Part II, 363 (1983). 
${ }^{3 T} \mathrm{~F}$. TROYON, T. GRLBER. H. SAUREMANN, S. SEMENZATO, S. SUCCCI. "MHD-Limits to Plasma Confinement," Plasma Phys. Controlled Fusion 26. 209 (1984).

${ }^{38}$ Y. C. SUN, D. E. POST, G. BATEMAN, and D. STOTLER, "Power Balance Calculations for CIT and Ignition Designs," Bull. Am. Phys. Soc. 32, 1921 (1987).

${ }^{39}$ C. Z. CHENG, H. P. FURTH, and A. H. BOOZER, "MHD Stable Regime of the Tokamak," Plasma Phys. Controlled Fusion 29, 351 (1987).

${ }^{*} \mathrm{~N}$. A. UCKAN and J. SHEFFIELD, "A Simple Procedure for Establishing Ignition Conditions in Tokamaks," in Tokumak Startup - Problems and Scenarios Related to the Transient Phases of Ignited Tokamak Operation (Plenum, New York, 1986), 45.

${ }^{41}$ M. W. PHILLIPS, Grumman Space Systems Division, private communication.

42J. MANICKAM, N. POMPHREY, and A. M. M. TODD, "Ideal MHD Stability Properties of Pressure-Driven Modes in Low Shear Tokamaks," Nucl. Fusion 27, 1461 (1987).

${ }^{43}$ G. REWOLDT, " $\alpha$-Particle Effects on High-n Instabilities in Tokamaks," Bull. Am. Phys. Soc. 32, 1771 (1987).

${ }^{44}$ J. D. CALLEN, J. P. CHRISTLANSEN, J. G. CORDEY, P. R. THOMAS and K. THOMSEN, "Modelling of Temperature Profiles and Transport Scaling in Auxiliary Heated Tokamaks," Nucl. Fusion 27, 1857 (1987).

${ }^{45} Y$. SHIMOMURA and K. ODAJIMA, "Empirical Scaling of Incremental Energy Confinement Time of L-Mode Plasma and Commests on Improved Confinement in Tokamaks, ${ }^{,}$Comments on Plasma Physics and Controlled Fusion 10,207 (1987).

${ }^{46}$ C. E. SINGER, G. BATEMAN, and D. P. STOTLER, "Boundary Conditions for H-Mode Simulations in Tokamaks," Bull. Am. Phys. Soc. 32, 1917 (1987). 
${ }^{4} J$ JET Team, "Latest Results from JET," Bull. Am. Phys. Soc. 32, 1711 (1987).

${ }^{43}$ D. P. STOTLER and G. BATEMAN, "Sirnulations of a Density Limit in Radiation-Dominated Tokamak Discharges," Bull. Am. Phys. Soc. 32, 1918 (1987). 


\begin{tabular}{|l|l|c|}
\hline major radius (m) & $R_{0}$ & 1.75 \\
minor radius (m) & $a$ & 0.55 \\
elongation & $\kappa$ & 2.0 \\
triangularity & $\delta$ & 0.4 \\
toroidal field (T) & $B_{T}$ & 10.0 \\
plasma current (MA) & $I_{p}$ & 9.0 \\
\hline
\end{tabular}

Table 1: Flat-top parameters for CIT 


\section{Figures}

FIG. 1. Time dependence of various specified discharge parameters; units are given separately with each.

FIG. 2. Contours of constant toroidal flux at various times for the fast ramp case discussed in Sec. III.B (similar to simulation I).

FIG. 3. Time dependence of the ohmic, auxiliary, and alpha power for simulaition I (solid line). The dashed line represents a simulation in which helium ash is pumped out during the flat-top.

FIG. 4. Time dependence of the ohmic, auxiliary, and alpha power for simulation II.

FIG. $5 . \beta$ due to fast alpha particles in simulation $I$ as a function of time and plasma half-width.

FIG. 6. Global energy confinement time as a function of time for simulations I (dashed line) and II (solid line). The arrows indicate the times of pellet injection.

FIG. 7 . Electron density as a function of radial half-width and time for simulation I.

FIG. 8. Electron density as a function of radial half-width and time for simulation II.

FIG. 9. Ion temperature as a function of radial half-width and time for simulation I.

FIG. 10. Ion temperature as a function of radial half-width and time for simulation II.

FIG. 11. Current density as a function of radial half-width for four different simulations at (a) $t=3$. and (b) $t=7$. sec. Curves for the three different ramp schemes discussed in Sec. III.B and for simulation II are shown.

FIG. I2. Time evolution of $q_{\text {gad }}$ for three different plasma shape ramp schemes. The line patterns are as in Fig. 11; simulation II follows the same path as the fast ramp scheme. 
FIG. 13. Time evolution of the sawtooth mixing radius for three different plasma shape tamp schemes and simulation II. The line patterns are as in Fig. 11. There are no sawteeth between $t \simeq 1.5 \mathrm{sec}$ and $t \simeq 8 \mathrm{sec}$ for the fixed shape case.

FIG. 14. Typical plot of the contours of auxiliary power (solid lines) required to maintain steady state in CIT as a function of $\left\langle n_{e}\right\rangle$ and $\left\langle T_{e}\right\rangle$. We indicate also contours of constant $P_{\alpha}$ (dashed lines).

FIG. 15. Trajectories for simulation I without ash removal (solid circles) and with ash removal (open circles) in hydrogenic density vs. ion temperature space. Heuristic curves for the ignited equilibrium $\left(P_{\text {net }}=0\right)$ and marginal thermal stability contours $\left(d P_{\text {net }} /\left\langle T_{i}\right\rangle=0\right)$ are included to allow a qualitative comparison with Fig. 14. The contours are drawn so as to be consistent with the data from the two runs. 


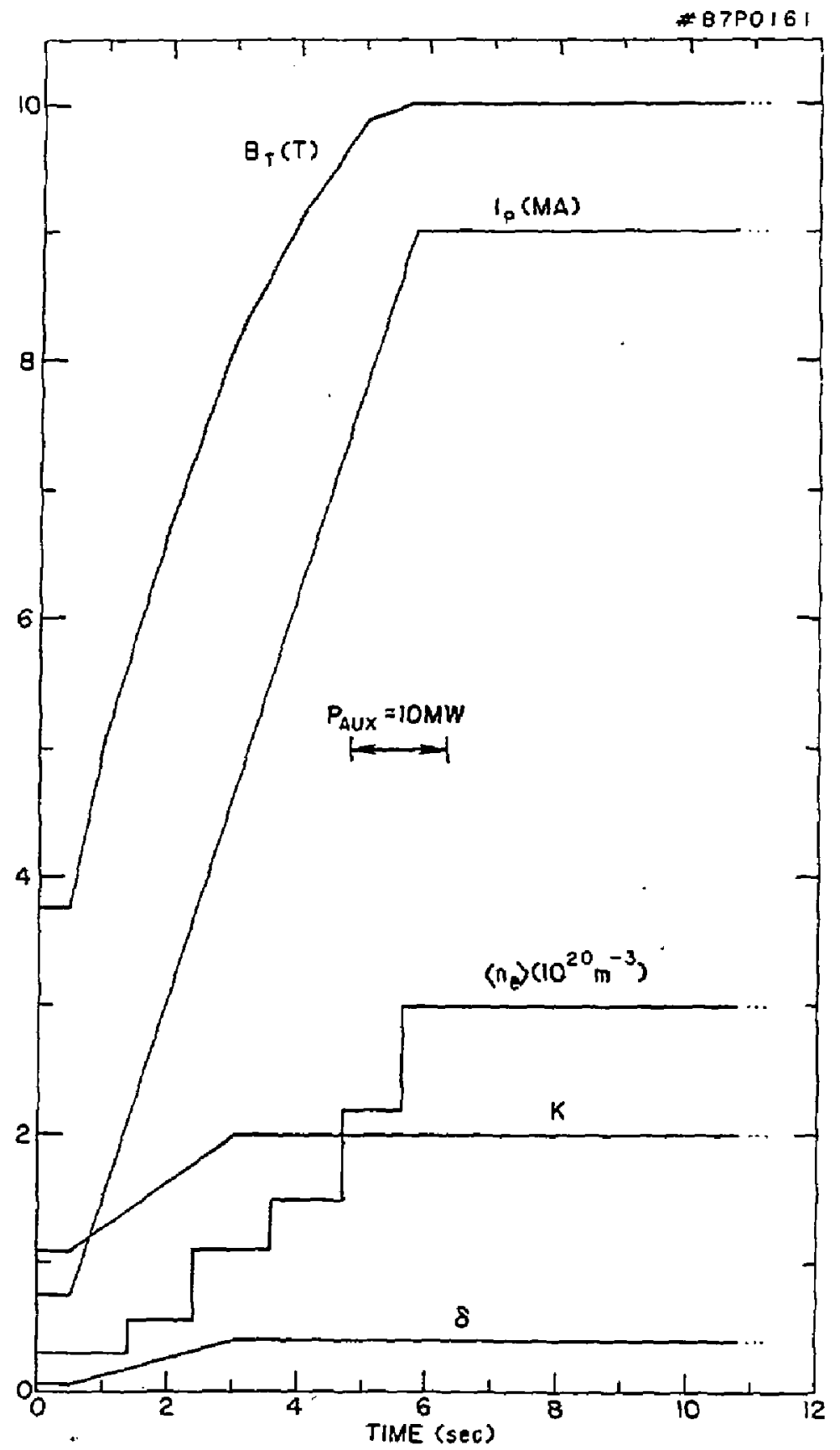

Fig. 1 

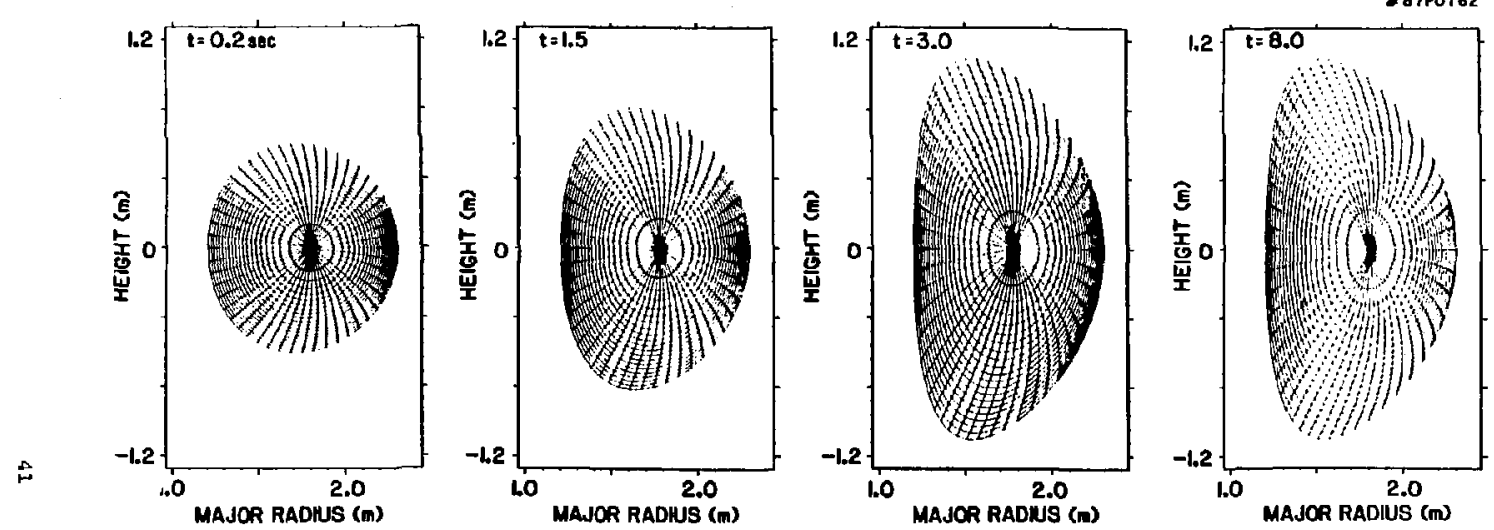

Fig. 2 
\#87PO163

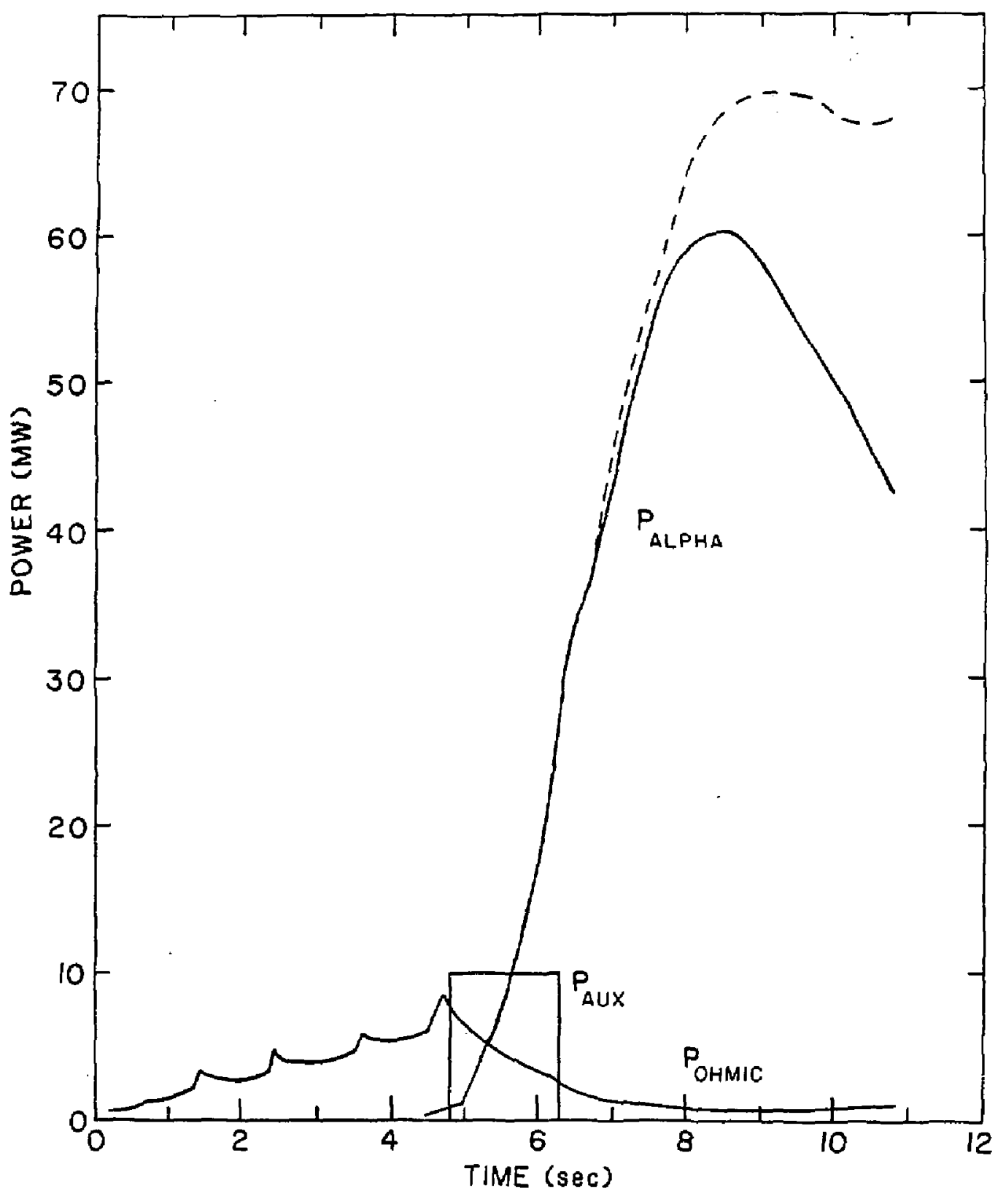

Fig. 3 


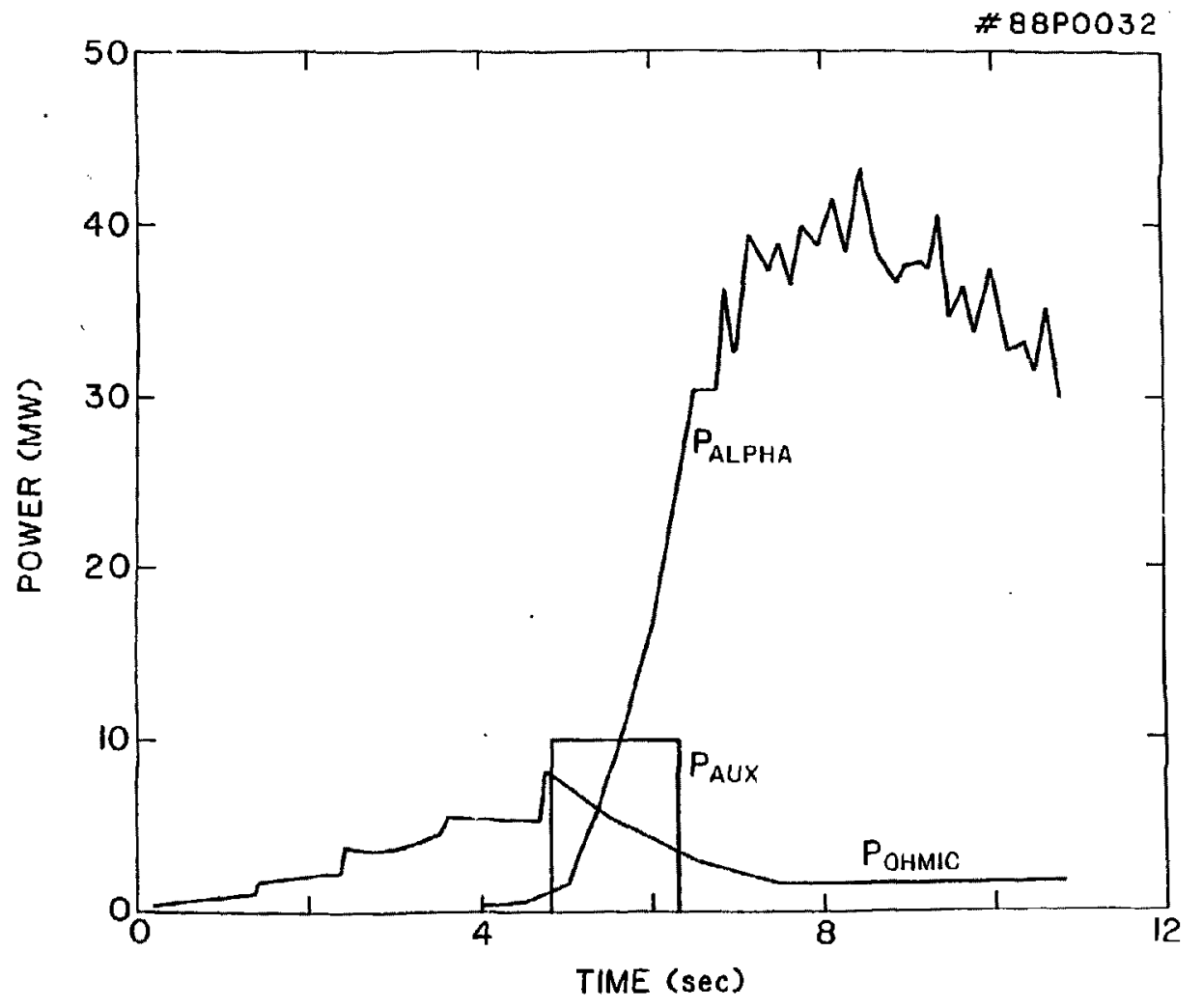




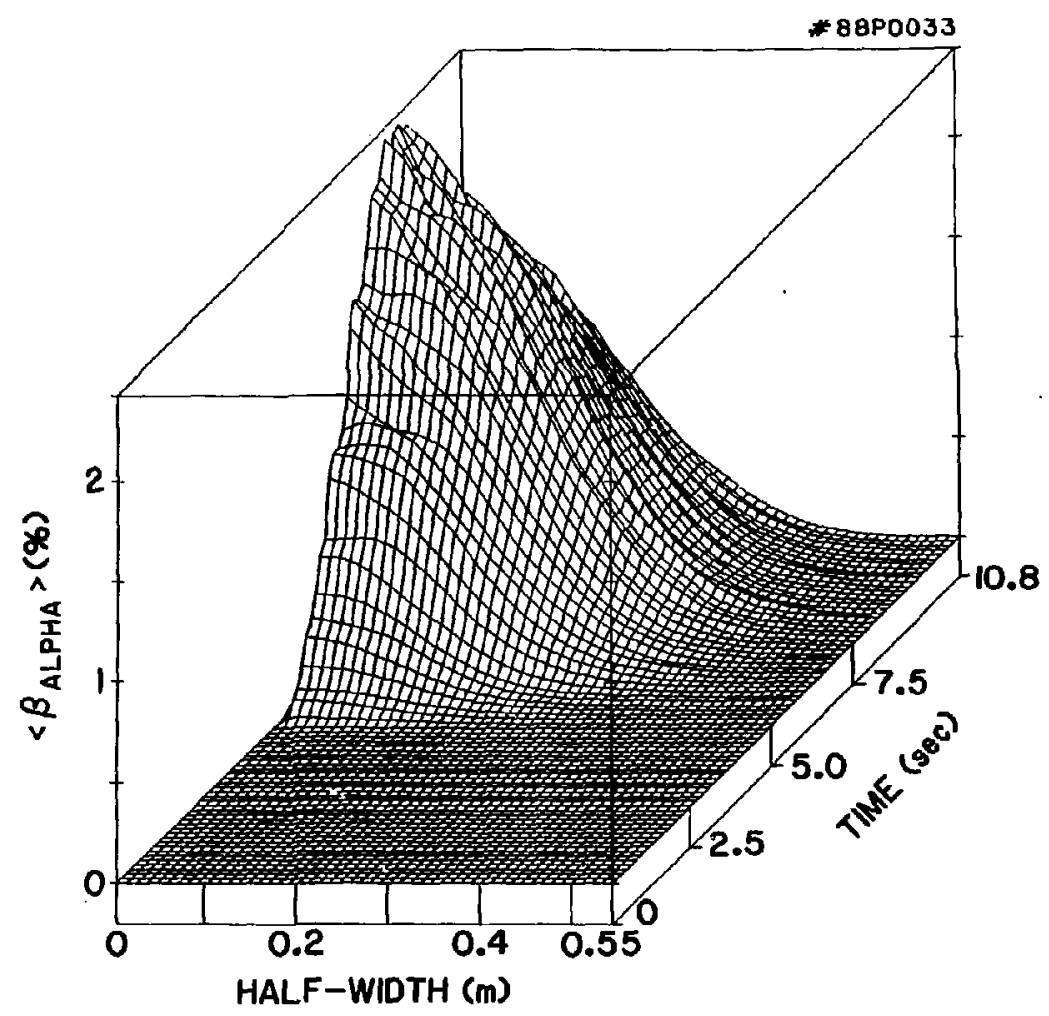

Fig. 5 


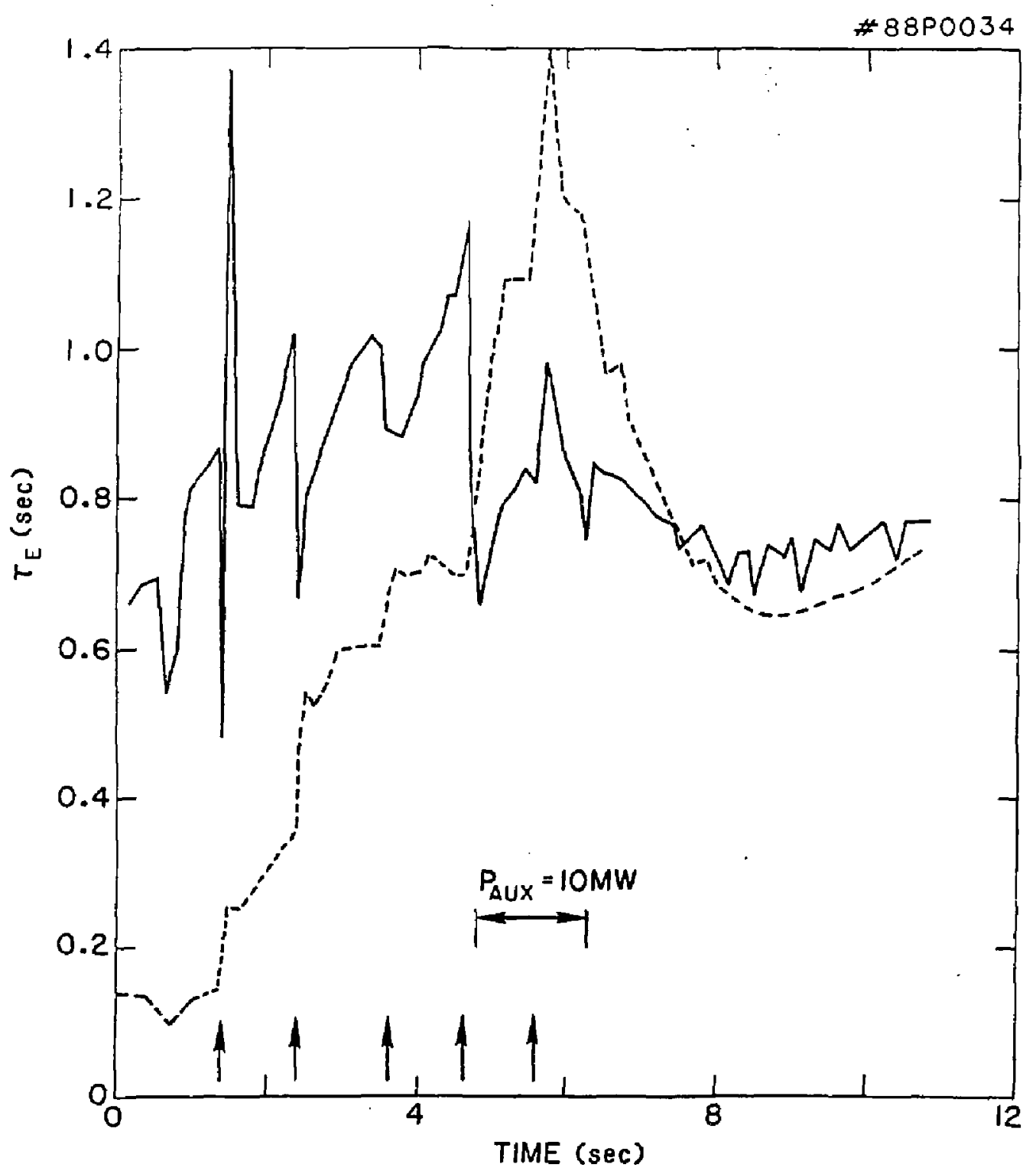

Fig. 6 


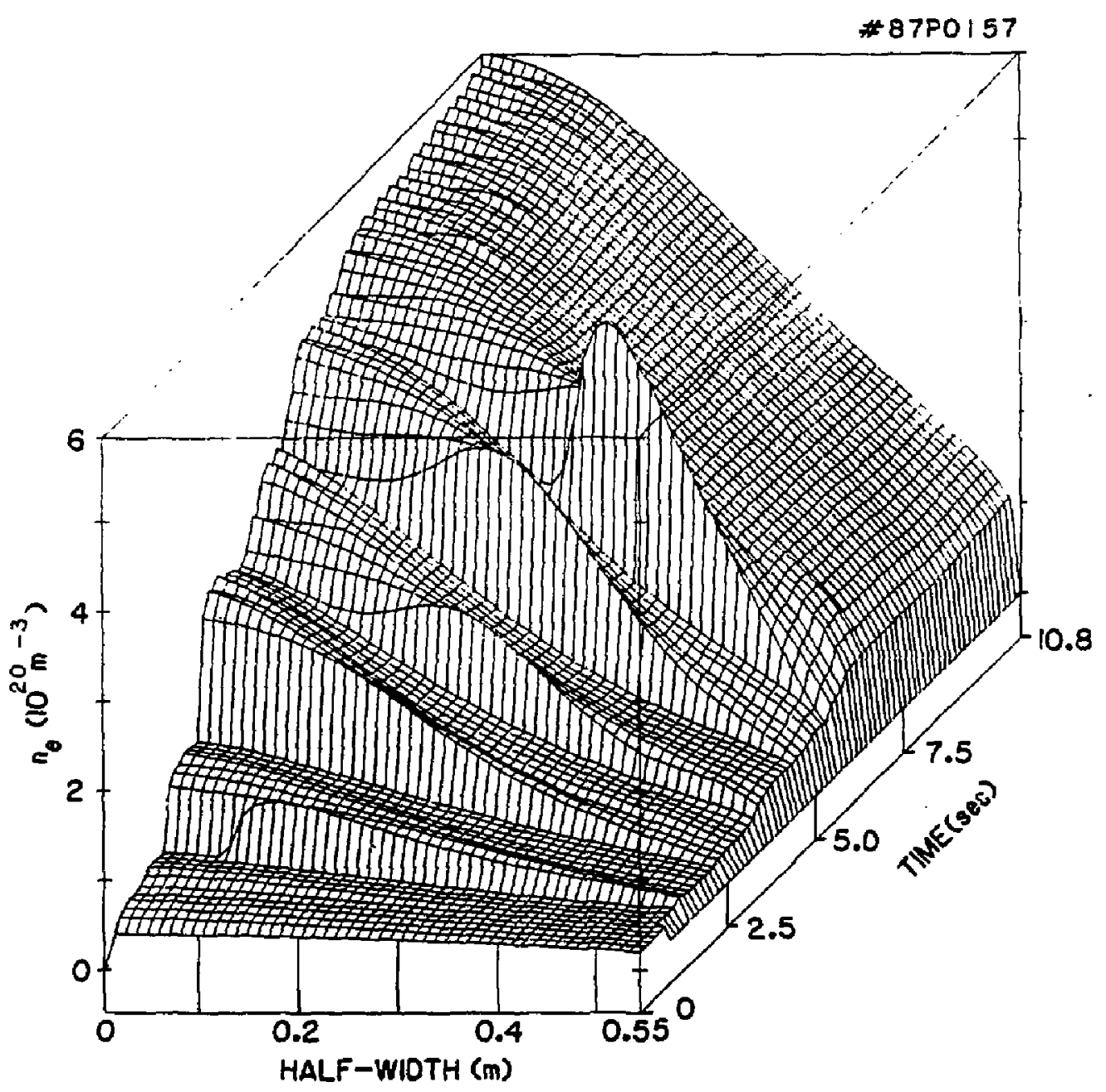

Eig. 7 


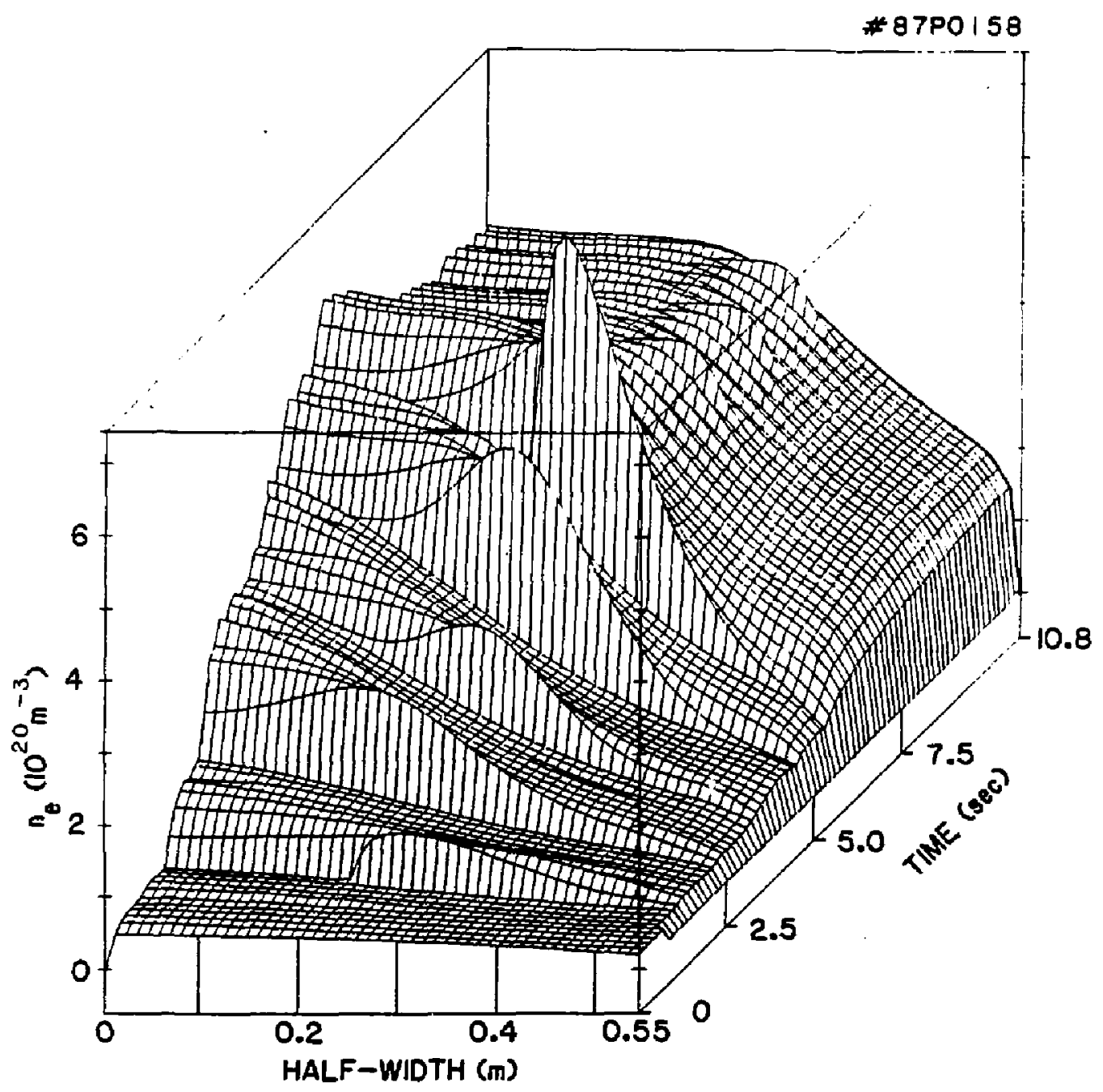

Fig. 8 


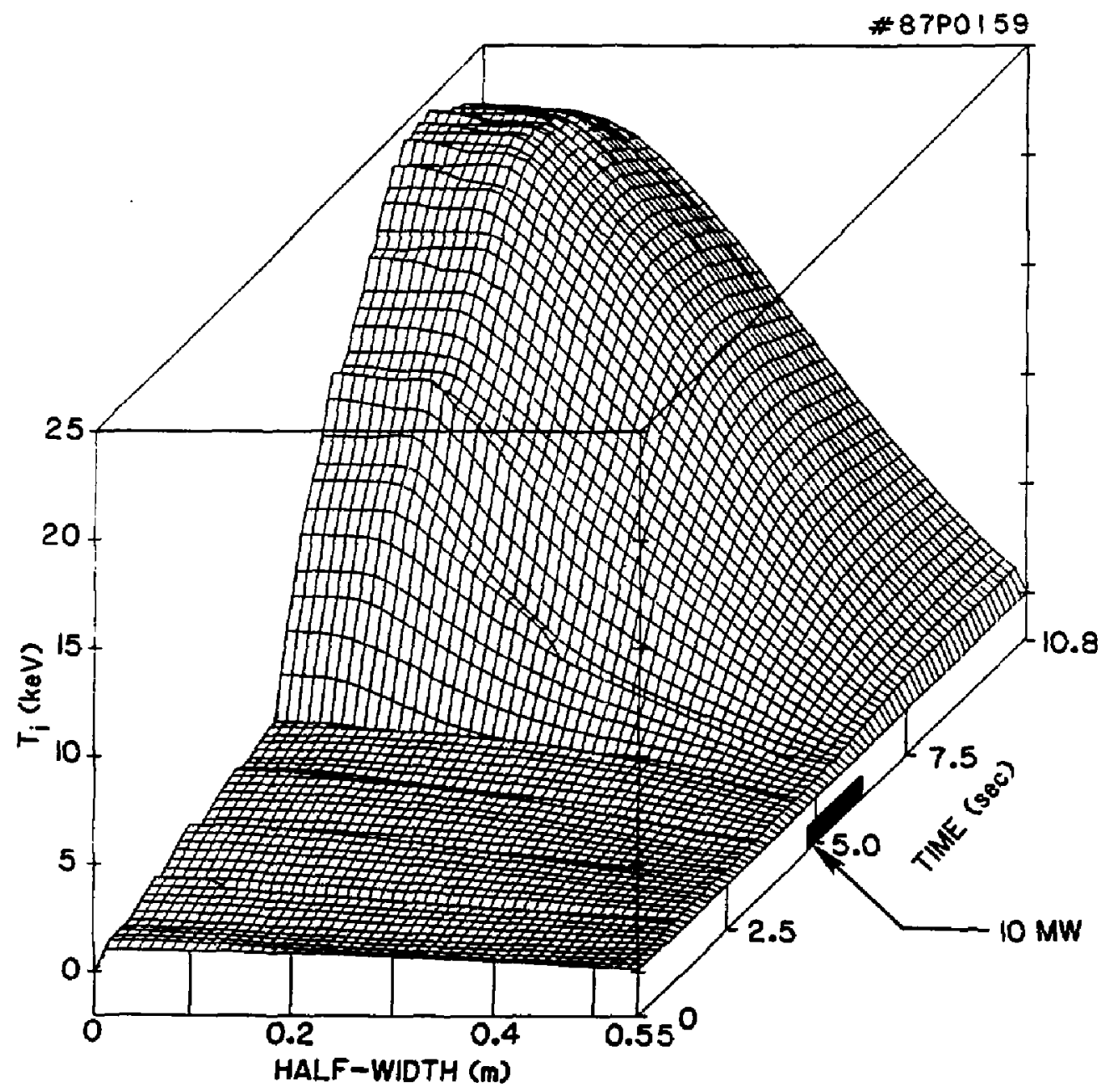

Fig. 9 


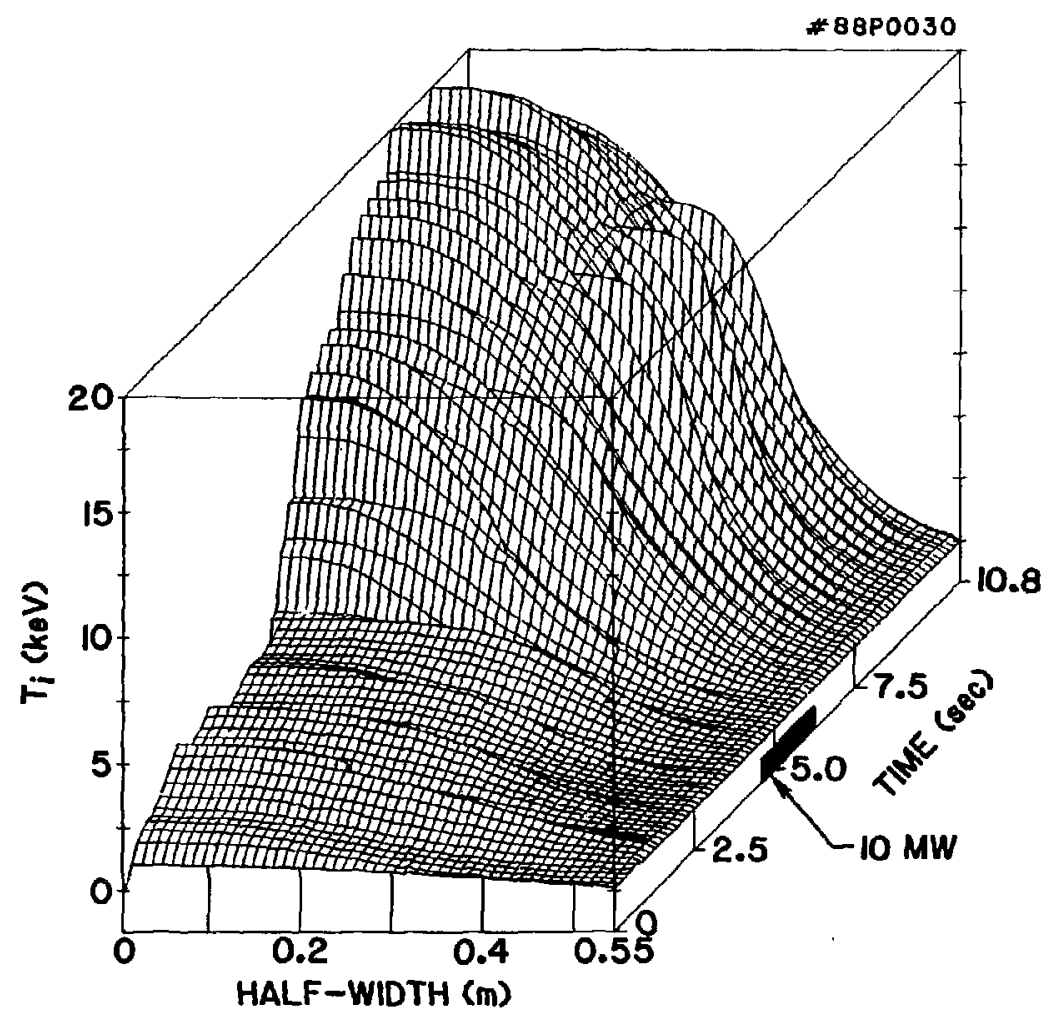

Fig. 10 

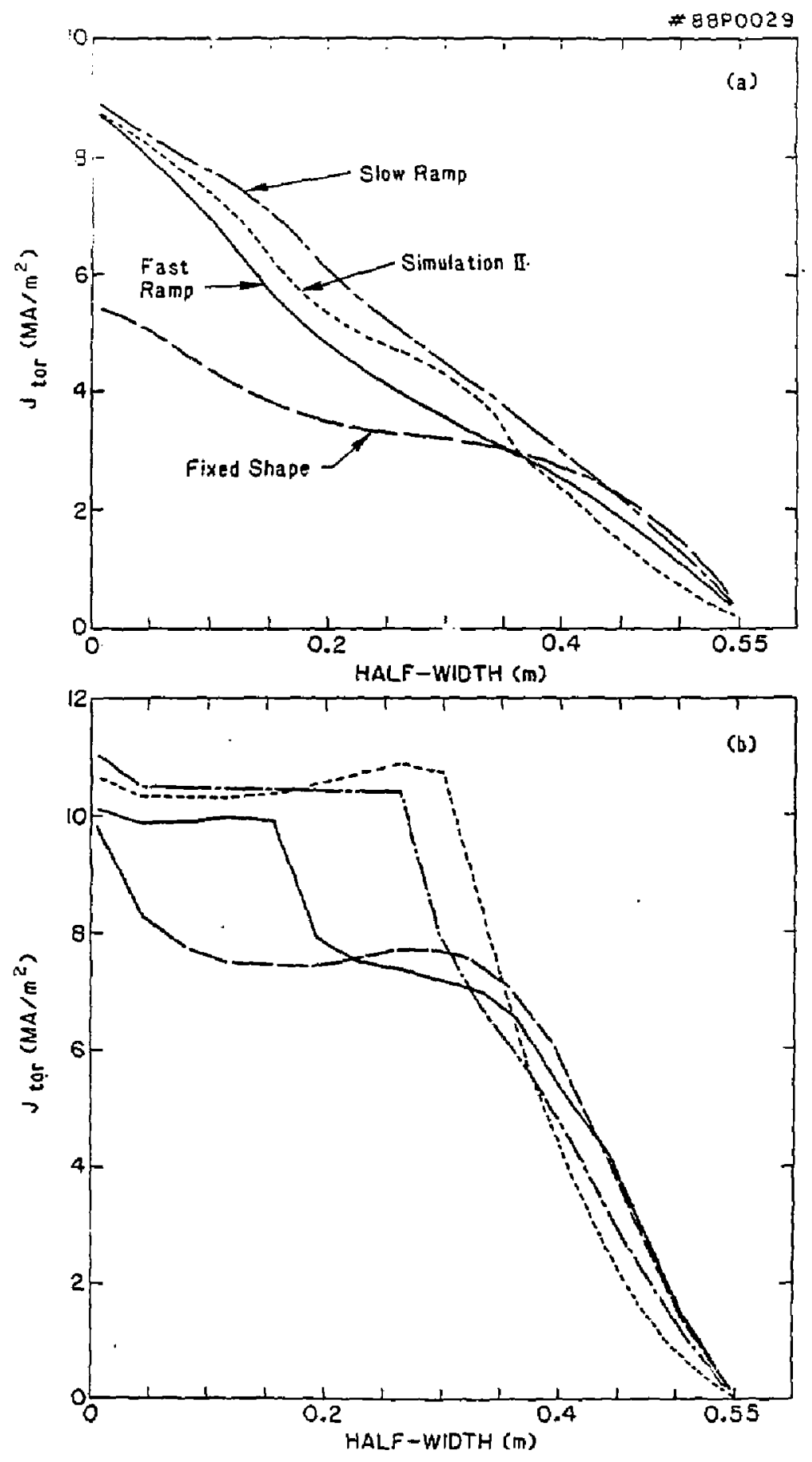

Fig. 11 


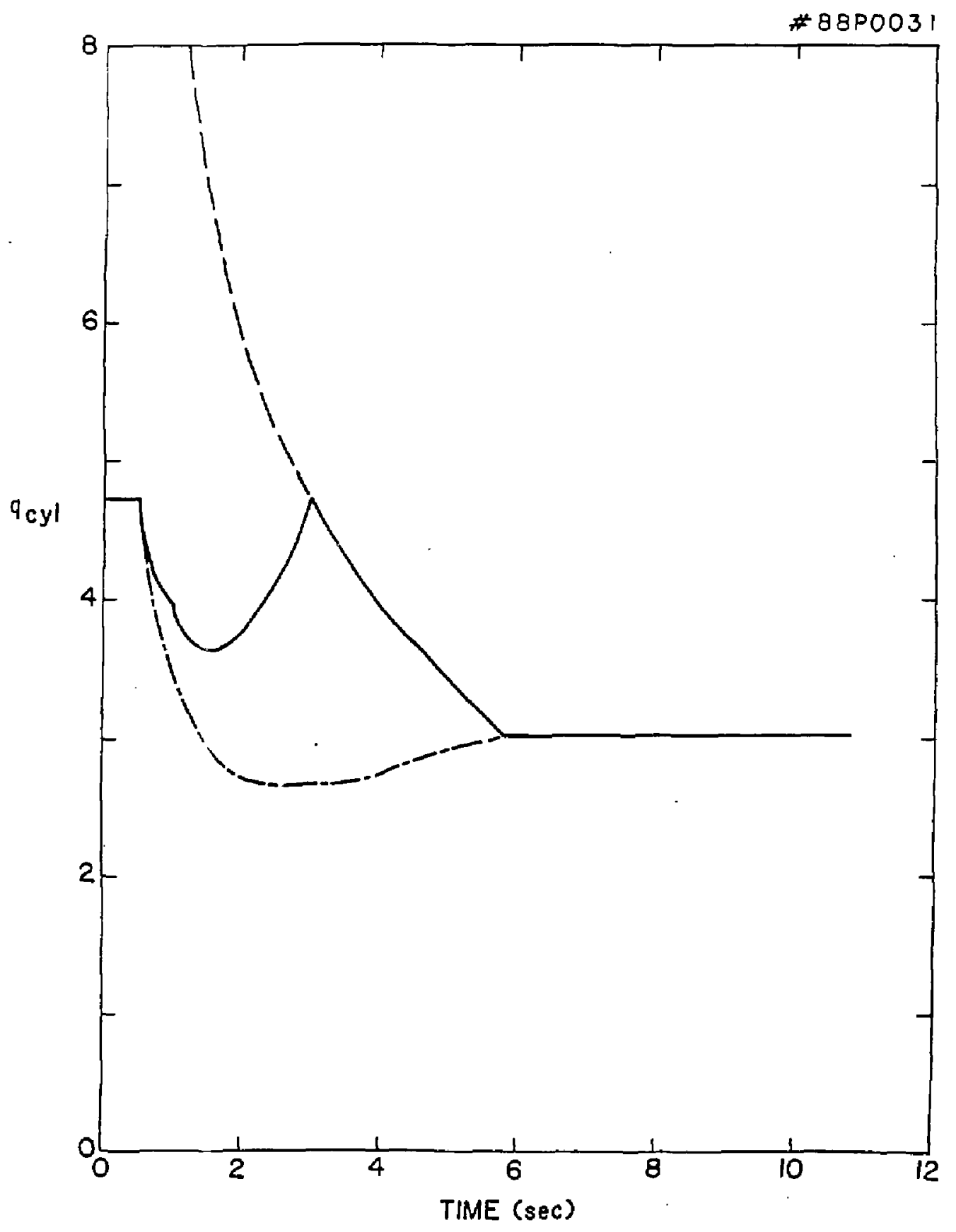

Fig. 12 


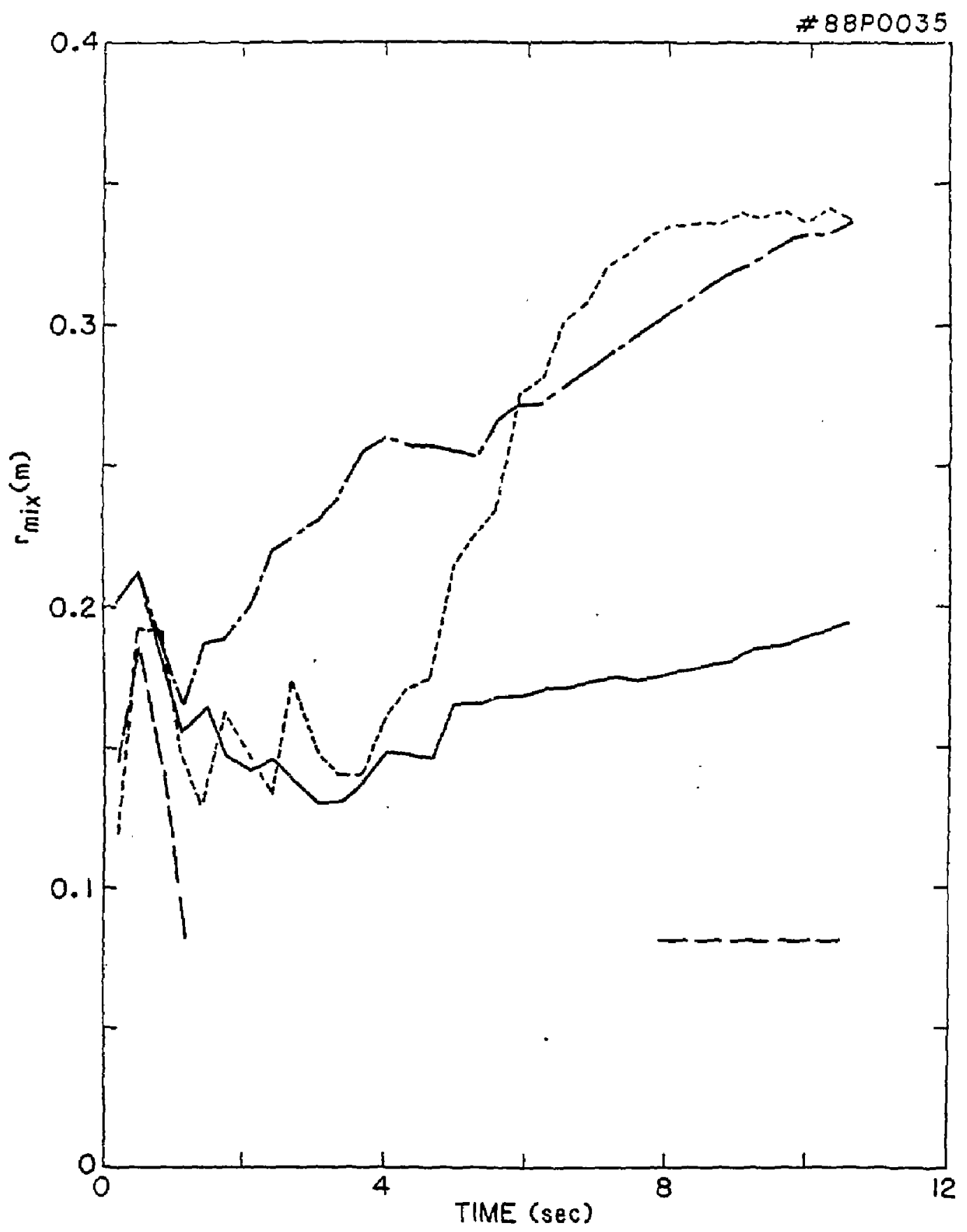

Fig. 13

52 


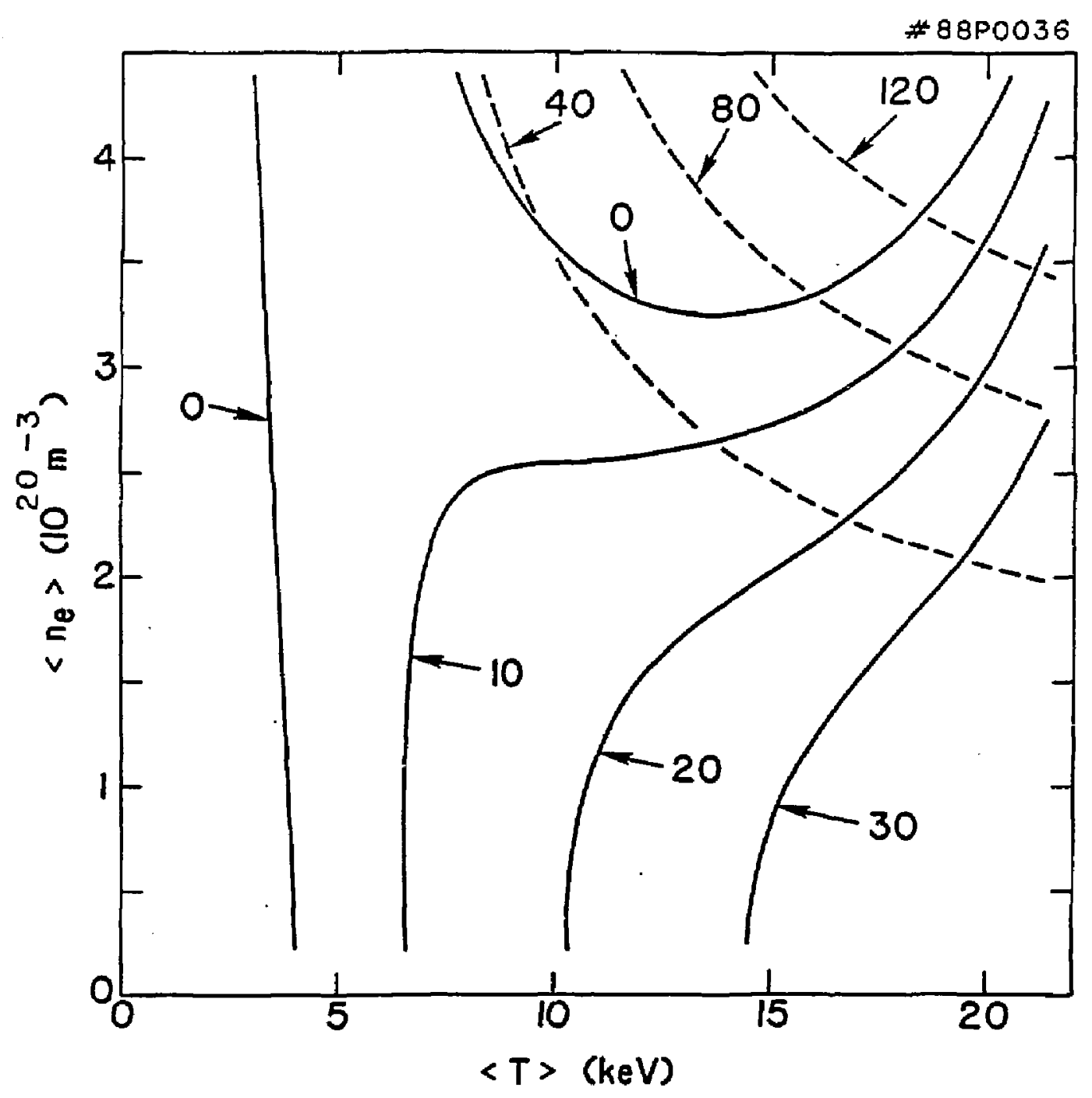

Fig. 14 


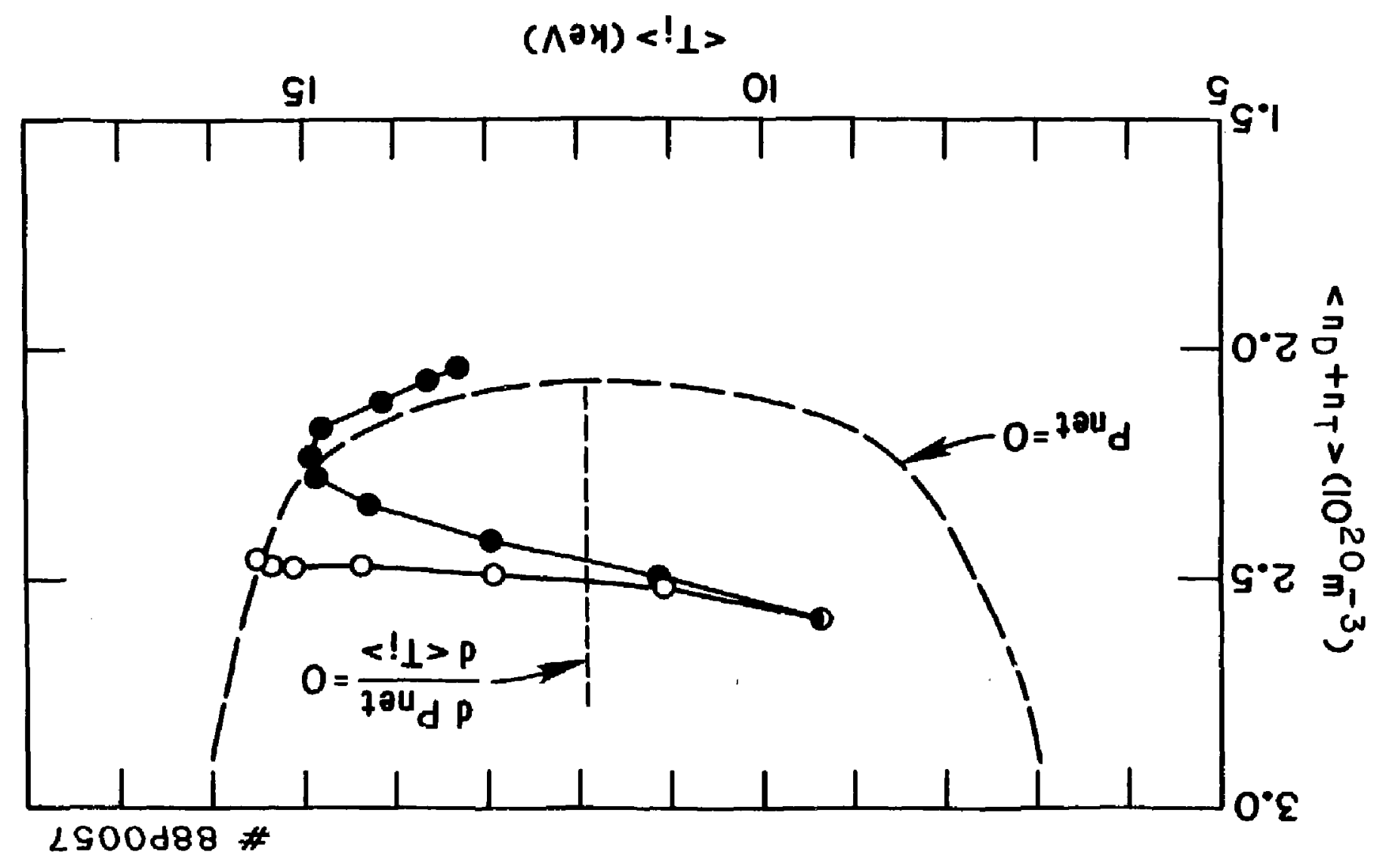


Dr. Frank J. Paoloni, Univ of wollongong, AUSTRALIA Prof. M.H. Brennan, Univ Sydney, AuSTRALiA

Plasma Research Lab., Australian Nat. Univ,., AlJSTRALIA

Prof. I.R. Jores, Flinders Univ., AuSTRALIA

Prot. F. Cap, Inst Theo Phys, AUSTRIA

Prof. M. Heindler, Institut fur Theoretische Physik, AUSTRIA

M. Coossens, Astronomisch Instituut, BELGIUM

Ecole Roysle Militalre, Lob de Phys PIasnas, BELGIun Comission-Eurogean, Dg-Xil Fusion Prog, BELGIuM

Prof, R. Boucique, Laboratorium voor Matuurkunde, BELGILM

Or. P.H. Sakanakg, Instltuto Fisica, GRAZIL

Instituto De Pesquisas Espaciasi-IAPE, ERAzIL

Dosuments Office, aromic Energy of Canada Limited, Cankom

Dr. M.P. Bachynski, MPe Technologias, Inc., CANADA

Dr. H.M. Skarsgard, University of Saskatcheman, CAMADA

Dr. H. Bernard, Univarsity of British Columbia, CANADA

Prot. J. Telchann, Univ. of Montreal, CANAOA

Prof. S.R. Sremivasan, University of Calgary, CANADA

Prof. Tudor W. Johnston, ImRS-Energie, CANADA

Or. C.R. James, Univ, of Alberta, CAMADA

Or: Poter Lukac, Komanskeho Univ, CZECHoslovakiA

The Librarian, Cuiha Laboratory. ENGLAND

The Librarian, Rutherford ADpleton Laboratory. ENGLAND

Mrs. S.A. Hutchinson, JET Library. ENGLAND

C. Mouttet, Lab. de Physique des Mili ieux Ionisés, france

J. Rodet, CEn/CADARACHE - Bat 506, FRAnCE

Univ, of loannina, Library of Physics Dept. Gefece

Dr. Tom Mual, Acadeny Bibliographic Ser., Hows KOns

Preprint Library, Hungar ian Acadeny of Sciences, HUNGaRY

Dr. B. Dasgupta, Sahe Inst of Nucl. Phys., IRDIA

Dr. P. Kan, Institute for Plasma Research, INDIA

Dr. Philip Rosenau, israel inst. Tech, ISRAEL

Librarian, Int'I Cir Theo Phys, ITALY

Prof. G. Rostagni, Univ Di Padova, ITALY

Miss Clelia De Pelo, Assoc EuRatoh-EneA, ITAly

Bibliateca, Instituto di Fisica del Plasme, I JALY

Dr. H. Yamato, Toshlba Res a Dev, JAPAN

Prof. I. Kawakeoi, Atomic Enargy Res. Instituto, JAPAN

Prof. Kyoji Nishikawo, Univ of Hiroshima, JAPAN

Direc. Dept. Large Tokemak Res. JAERI, JAPAN

Prof. Satoshi Itoh, Kyushu Univorsity, JAPAN

Research Info Center, Nagoya University, JAPAN

Prof. S. Tanaka, Kyoto University, JAPAN

Library. Kyoto University, JAPAN

Prof. Nobuyuki Inoue. University of Tokyo, JAPAN

S. Mori, JAERI, JAPAN

Librarian, Kores Advanced Enargy Ras. Institute, KOREA

Prof. D.I. Choi, Adv. Inst Sel \& Tech, KOREA

Prof, B,s. Liley, University of Haikato, WEH ZEALANO

Institute of Plasmo Physics, PEOPLE'S REPUBLIC Of CHINA

Librarian, Institute of Phys., PEOPLE'S REPUBLIC OF CHINA

Library, Tsing Hus University, PEOPLE'S REPUBLIC OF CHINA
2. Li, Southwest Inst. Physies, PEOPLE'S REPU⿴LIC OF C Prof. J.A.C. Cabral, Inst Superior Tecnico, PORTUGAL

Dr. Octavian Petrus, AL I CUzA University, ROMANIA

Dr. Johan de Villiers, Fusion studies, AEC, SO AFAICA

Prot. M.A. Hellberg, University of Natal, SO AFRICA

C.I.E.M.A.T, Fusion Div. Library, SPAIN

Or, Lennart Stenllo, University of UMEA, SWEDEN

LIbrary, Royal Inst Tech. SWEDEN

Prof. Hans wilhelnson, Chalmers Un iv Tech, SWEDEN

Centro Phys des Plasnas, Ecolo Polytech Fed, SwITZERLAi

Bibliotheok, fom-Inst Voor Plasma-fysica, THE NETHERLAi

Dr. D.D. Ryutor, Sibarian Acad Sel, USSR

Dr. G.A. El iseev, Kurchator Institute, USSR

Dr. Y.A. Glukhikh, Inst Electrophysical Apparatus, USSR

Dr. Y.T. Tolok, Inst. Phys. Tech. USSR

Dr. L.M. Kovrizhnykh, Institute Gen. Physics, USSR

Nuclear Ras. Establisnnent, Julich Ltd,. W. GERAMAY

Bibliothek, Inst. Fur Plasmatorschung, W. GERMANy

Dr. K. Schindler, Ruhr Universitat Bochum, W. GEFWANY

ASDEX Reading Rm, IPP/Max-PIanck-lnstitut fur

Plasmaphysik, W. Gepahy

Librarian, Mastalanck Institut, W. GEPaMAYY

Prof. R+K. Janev, Inst Phys, rugoslavia 